\title{
21st International Symposium on Transportation and Traffic Theory
}

\section{Data-driven linear decision rule approach for distributionally robust optimization of on-line signal control}

\author{
Hongcheng Liu ${ }^{a}$, Ke Han ${ }^{b, *}$, Vikash Gayah ${ }^{\mathrm{c}}$, Terry Friesz ${ }^{\mathrm{a}}$, Tao Yao ${ }^{\mathrm{a}}$ \\ ${ }^{a}$ Department of Industrial and Manufacturing Engineering, Pennsylvania State University, University Park, PA 16802, USA \\ ${ }^{b}$ Department of Civil and Environmental Engineering, Imperial College London, London SW7 2BU, UK \\ ${ }^{c}$ Department of Civil and Environmental Engineering, Pennsylvania State University, University Part, PA 16802, USA
}

\begin{abstract}
We propose a two-stage, on-line signal control strategy for dynamic networks using a linear decision rule (LDR) approach and a distributionally robust optimization (DRO) technique. The first (off-line) stage formulates a LDR that maps real-time traffic data to optimal signal control policies. A DRO problem is then solved to optimize the on-line performance of the LDR in the presence of uncertainties associated with the observed traffic states and ambiguity in their underlying distribution functions. We employ a datadriven calibration of the uncertainty set, which takes into account historical traffic data. The second (on-line) stage implements a very efficient linear decision rule whose performance is guaranteed by the off-line computation. We test the proposed signal control procedure in a simulation environment that is informed by actual traffic data obtained in Glasgow, and demonstrate its full potential in on-line operation and deployability on realistic networks, as well as its effectiveness in improving traffic.

(C) 2015 The Authors. Published by Elsevier B.V. This is an open access article under the CC BY-NC-ND license

(http://creativecommons.org/licenses/by-nc-nd/4.0/).

Selection and peer-review under responsibility of Kobe University

Keywords: on-line signal control; LWR model; linear decision rule; distributionally robust optimization; data-driven modeling
\end{abstract}

\section{Introduction}

Signalized intersections are often the most restrictive bottlenecks in urban traffic networks. For this reason, urban traffic control strategies tend to focus on the operation of individual intersections (e.g., see Miller, 1963; Robertson and Bretherton, 1974; Guler and Cassidy, 2012; Gayah and Daganzo, 2012). And, operations at individual signalized intersections are often used to describe traffic conditions in urban environments (Highway Capacity Manual, 2000).

Optimizing signal timing plans at these locations has been the subject of much research. The optimization procedure employed typically takes on one of two forms: 1) those developed based on historical information, often referred to as fixed timing plans; and, 2) those that are fully responsive to current traffic conditions, often referred to as adaptive (or on-line) signal controls. The former case is less efficient, as signal timings are not able to adapt to naturally occurring variations in traffic flows. This can result in significant periods of queuing and delays during large fluctuations in traffic flows. In the latter case, signal parameters such as cycle lengths and splits between competing movements are determined based on real-time traffic data.

\footnotetext{
* Corresponding author. Tel.: +44-020-7594-5682 ; fax:+44-020-7594-6102.

E-mail address: hq15143@psu.edu; k.han@imperial.ac.uk; gayah@engr.psu.edu; tfriesz@psu.edu; taoyao@psu.edu
} 
Real-time signal controls react to unpredicted traffic conditions in a more timely fashion and are more robust to local network disruptions when compared to fixed timing plans. From an optimization point of view, a well-defined function is required to relate the signal parameters to specific objective being optimized. Specific objectives in the literature include the minimization of vehicle delay (Zhang et al., 2010; Sun et al., 2006), minimization of passenger delay (Christofa and Skabardonis, 2011), minimization of number of stops (Lucas et al., 2000), and maximization of total throughput (Chang and Sun, 2004; Han et al., 2014). However, analytical formulations are not always readily available for these objective functions. Furthermore, the objectives often tend to be non-linear functions of the signal parameters and the traffic flow variables, as in the case of traffic delays or vehicle stops. The resulting optimization problem usually becomes a computationally complex non-linear and/or non-convex mathematical program that is difficult to solve exactly. Instead, many real-time signal optimization procedures are developed using heuristic approaches such as genetic algorithm and fuzzy logic (e.g., Foy et al., 1992; Chiu and Chand, 1993; Ceylan and Bell, 2004; Murat and Gedizlioglu, 2005). Unfortunately, solutions obtained in this way are usually inexact and suboptimal.

In this paper, we propose a novel linear decision rule (LDR) approach for responsive signal control, which takes into account both historical and real-time traffic data. In addition, we employ a distributionally robust optimization (DRO) formulation and a data-driven calibration of the underlying uncertainty set to handle both within-day and day-to-day variations in traffic flow. The proposed methodology involves a two-stage operation of signal control. In the first (off-line) stage, a distributionally robust optimization (DRO) problem is formulated based on a linear decision rule (LDR). The goal of this formulation is to optimize the on-line performance of the decision rule with a range of uncertainties associated with the underlying distribution of the network flows, and to ensure its sound performance even with the worst-case scenario. Adding novelty to the methodology is a data-driven procedure for the calibration of the uncertainty set with arbitrary number of samples and confidence level. Furthermore, we propose two solution schemes for the off-line computation: one based on a mixed integer linear program, and the other based on a metaheuristic search. In the second (on-line) stage, the optimized LDR obtained from the first stage is used to convert real-time information into signal control parameters in a timely fashion. Moreover, the performance of such a simple decision rule is guaranteed by virtue of the DRO performed off line. The proposed signal decision architecture takes into account average traffic pattern while capturing its stochastic component. The proposed method combines solutions obtained from mathematical programs with a practical decision rule that can be fully realized in real time. It also resorts to the theory of distributionally robust optimization and data-driven calibration of the uncertainty set, and thus guarantees optimality under various stochastic circumstances. One of the primary benefits of this approach is that all of the expensive computations are performed off line, making real-time control easily implementable.

Our proposed model significantly differs from existing ones in the literature. First of all, our model incorporates a linear decision rule approach in determining the optimal signal strategy as a response to the real-time traffic states. This approach assumes that the responsive signal control is a linear mapping of the observed traffic states and computes its optimal parameters to improve the system performance. In comparison with the signal strategies based on historical and/or real-time information in the literature, our approach addresses the sophistication of a traffic system with a mathematical program that incorporates the Lighthill-Whitham-Richards traffic flow model (Lighthill and Whitham, 1955; Richards, 1956). This enables the model to capture complex traffic behaviors including physical queuing, shock waves, and spillbacks. Moreover, both the on-and-off signal model and the continuum signal model (Han et al., 2014) are considered and incorporated. ${ }^{1}$ In comparison with the current modeling approaches that rigidly preset the signal sequences for an assumed incoming flow, the linear decision rule determines the optimal signal timing plan in response to both real-time and historical traffic states and is, therefore, potentially more flexible, robust, and responsive. Another advantage of this approach is that the computation of the optimal decision rule can be conducted in an off-line environment and thus significantly reduces the real-time computational efforts.

Secondly, the proposed model allows the traffic network flows to be uncertain and mitigates the impacts of uncertainty with a distributionally robust optimization (DRO) procedure. DRO bridges between two popular classes of optimization techniques under uncertainty: stochastic programming (SP) and robust optimization (RO). The former

\footnotetext{
${ }^{1}$ The continuum signal model approximates the average effect of the periodic and on-and-off signal controls at an intersection by replacing the binary controls with a continuous parameter (the green ratio). The continuum signal model predicts the aggregate traffic dynamics that exist at signalized intersections and networks without relying on detailed information of vehicle movements and signal phasing plans, and thus entails improved computational efficiency.
} 
requires the exact knowledge of the underlying probability distribution. When such knowledge is not available, SP may perform poorly (Bertsimas et al., 2011). In contrast to SP, RO allows total absence of distribution information, and seeks to optimize in the worst-case scenario among all possible realizations of uncertainty (Goh and Sim, 2010). However, it has been criticized to be too conservative in some cases. Seeking to strike a balance between the specificity of SP and the conservatism of RO, the DRO approach adopted in this paper allows the distribution functions to be defined ambiguously and optimizes the expected objective with the most adversarial distribution. The resulting signal decisions can provide a sound system performance in the presence of uncertainty and inexactness in the distribution information.

Thirdly, to capture the set of candidate distribution functions, we employ a data-driven approach proposed by Bertsimas et al. (2013), which leverages existent statistical learning techniques in constructing the uncertainty set based on historical data available at hand. Specifically, we employ the Kolmogorov-Smirnov test to identify the set of distributions that contains the true distribution with a tunable probability guarantee. To our knowledge, this is the first mathematical programming model for signal control that incorporates a data-driven approach to capture uncertainties of the traffic system.

Last but not least, most existing on-line signal control strategies tend to have localized effect in that they collect and process data at a local region, often involving one or two signalized intersections, and implement controls that aim to optimize local traffic. These distributed controls often fail to achieve global optimality. In contrast, the proposed control strategy integrates historical and real-time data on the network-wide level, and is based on an off-line global optimization procedure. Thus, it is more likely to achieve global improvement of the network performance.

Two solution schemes are proposed for the off-line computation. The first one is an exact method based on a mixed integer linear program (MILP). As we demonstrate in Section 3, the LDR-based distributionally robust optimization, which is effectively a bi-level program, can be reduced to a single-level problem with mixed integer linear constraints by employing a dual formulation and a finite sampling approach. The second solution method is based on a metaheuristic search. This solution scheme is applicable to a range of problems with large spatial-temporal size, rather general junction type and signal specifications, a wide range of traffic network dynamics (other than the LWR), and a variety of network performance measures. It can even be easily integrated with commercial traffic simulation softwares (see Section 4.2). Both solution methods are tested on a real network in west Glasgow.

The rest of this paper is organized as follows. Section 2 describes in full mathematical detail the main architecture of the proposed two-stage signal control strategy, along with a data-driven process for constructing the uncertainty set. Section 3 presents a mixed integer linear program (MILP) formulation of the off-line problem, based on a dual approach and a finite sample approximation. Section 4 proposes a metaheuristic approach for solving the off-line problem, which complements the MILP and is suitable for larger and more realistic problems. Section 5 tests both MILP and metaheuristic implementations of the proposed on-line signal control on a real sub-network in Glasgow. Finally, Section 6 offers some concluding remarks.

\section{Linear decision rule (LDR) for on-line signal control}

This section describes in detail the LDR for the implementation of real-time, responsive signal control on an arbitrary network. Specifically, we stipulate a linear mapping between the observed system states and the signal control parameters. Coefficients and parameters of such a linear mapping are undetermined at this stage, and will be the subject of a distributionally robust optimization (DRO) procedure to be later discussed. The goal of this DRO procedure is to ensure the performance of the resulting responsive signal control in any realization of the stochastic inflow profile of the network, including the worst-case scenario.

\subsection{Deterministic formulation of the $L D R$}

We begin with the deterministic formulation of the LDR. Consider an arbitrary network with multiple intersections. Denote by $\mathbb{L}_{i n}$ the set of incoming links of the network under consideration, and let $\mathbb{L}_{s}$ be the set of links that are controlled by traffic signals. Define, for each link $i \in \mathbb{L}_{i n}$, its inflow $\bar{q}_{i}^{t}$ where $1 \leq t \leq N$ indicates the discrete time step. We then define the network inflow vector $\boldsymbol{q}_{i n} \doteq\left(\bar{q}_{i}^{t}: 1 \leq t \leq N, i \in \mathbb{L}_{i n}\right)$, which contains information on the inflows of the network for the entire time horizon. Moreover, we let $Q_{i n}$ be the set of all possible network inflow 
vectors. Clearly, we have that $Q_{i n} \subset \mathbb{R}_{+}^{\left|\mathbb{I}_{i n}\right| \times N}$. $Q_{i n}$ is viewed as the input set of our linear mapping. On the other hand, the output set, which contains signal control parameters, is defined as follows. We distinguish between the continuum signal control and the on-and-off signal control; for the former the control set is $\mathcal{U}_{1} \doteq[0,1]^{\mathbb{L}_{s} \mid \times N}$; for the latter the control set is $\mathcal{U}_{2} \doteq\{0,1\}^{\left|\mathbb{L}_{s}\right| \times N}$.

Remark 1. The control sets $\mathcal{U}_{1}$ or $\mathcal{U}_{2}$ should be refined with additional constraints/requirements at the signal junctions; examples include upper and lower bounds on the green/red time, fixed/dynamic signal cycles and splits, pedestrian and all-red phases, and signal coordination. As we shall show later, these considerations can be easily incorporated into our formulation. Therefore, without loss of generality we continue to use notations $\mathcal{U}_{1}$ and $\mathcal{U}_{2}$ to represent respectively the sets of feasible continuum and on-and-off signal controls, noting that they consist of signal control parameters that serve any practical purpose and comply with real-world requirements.

We introduce the following abstract network performance measure, which is interpreted as a mapping

$$
\Phi: Q_{i n} \times \mathcal{U}_{1} \rightarrow \mathbb{R} \quad \text { or } \quad \Phi: Q_{i n} \times \mathcal{U}_{2} \rightarrow \mathbb{R}
$$

Some examples of such a performance measure include network delay, network throughput, emission rate, and accident-related risk index. Obviously the network performance depends on the network inflows (indicated by $Q_{i n}$ ) and the signal controls (indicated by $\mathcal{U}_{1}$ or $\mathcal{U}_{2}$ ). Throughout the rest of this paper we will, without loss of generality, always assume that $\Phi$ is subject to minimization.

Fix any natural number $T \geq 1$, we assume that the LDR is applied at time $t$, with input given by the most recent $T$ observations of the incoming flows, i.e., with network inflow data at times $t-T, t-T+1, \ldots, t-1$. As such, the decision-making process can possess some memory of the near past, and make use of those data as they will affect the system in the near future. Given these inflow data for $T$ consecutive time intervals, we introduce linear coefficients $A \doteq\left(a_{l, i}^{t}: 1 \leq t \leq T, l \in \mathbb{L}_{i n}, i \in \mathbb{L}_{s}\right)$ and $b \doteq\left(b_{i}: i \in \mathbb{L}_{s}\right)$, and write

$$
\mu_{i}^{t} \doteq \sum_{l \in \mathbb{L}_{i n}} \sum_{j=\max \{1, t-T\}}^{t-1} \bar{q}_{l}^{j} \cdot a_{l, i}^{j-t+T+1}+b_{i} \quad \forall i \in \mathbb{L}_{s}, \quad \forall 1 \leq t \leq N
$$

We then form the vector $\mu \doteq\left(\mu_{i}^{t}: 1 \leq t \leq N, i \in \mathbb{L}_{s}\right)$, which is the collection of "preliminary" signal controls obtained directly by an affine transformation with coefficients $A$ and $b$. However, such a control vector in general does not satisfy various constraints associated with the real-world signal control. Thus we project $\mu$ onto the set of feasible signal controls. With some abuse of notation, we re-write (1) in a compact form as $\mu=A \cdot \boldsymbol{q}_{i n}+b$, and define the feasible signal control variables as

$$
\eta=\mathcal{P}_{\mathcal{U}_{1}}\left[A \cdot \boldsymbol{q}_{\text {in }}+b\right] \quad\left(\mathrm{LDR} \text { for continuum) } \quad \text { or } \quad u=\mathcal{P}_{\mathcal{U}_{2}}\left[A \cdot \boldsymbol{q}_{\text {in }}+b\right] \quad\right. \text { (LDR for on-and-off) }
$$

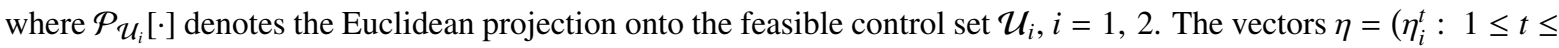
$\left.N, i \in \mathbb{L}_{s}\right)$ and $u=\left(\eta_{i}^{t}: 1 \leq t \leq N, i \in \mathbb{L}_{s}\right)$ are the feasible continuum and on-and-off control variables, respectively. Each $\eta_{i}^{t}\left(\right.$ or $u_{i}^{t}$ ) is the continuum (or on-and-off) signal decision for link $i \in \mathbb{L}_{s}$ at time $t^{2}$.

Given a network inflow vector $\boldsymbol{q}_{i n}$, the LDR (2) obviously defines a mapping from the pair $(A, b)$ to the control $\eta \in \mathcal{U}_{1}$ or $u \in \mathcal{U}_{2}$; and such a mapping is written as $\eta=U\left(A, b ; \boldsymbol{q}_{\text {in }}\right)$ or $u=U\left(A, b ; \boldsymbol{q}_{\text {in }}\right)$. There clearly exists a best choice $\left(A^{*}, b^{*}\right)$ such that the corresponding signal control $U\left(A^{*}, b^{*} ; \boldsymbol{q}_{\text {in }}\right)$ yields the best network performance among all other choices of $(A, b)$. Finding such $\left(A^{*}, b^{*}\right)$ amounts to the following mathematical program:

$$
\min _{A, b} \Phi\left(\boldsymbol{q}_{i n}, U\left(A, b ; \boldsymbol{q}_{i n}\right)\right)
$$

subject to constraints specified by the underlying traffic network model (such as the LWR model). (3) defines the deterministic version of the optimal LDR problem for on-line signal control. It has the following appealing features:

\footnotetext{
${ }^{2}$ In general the signal split parameter $\eta_{i}^{t}$ should remain constant for the duration of at least a full cycle in order for the continuum signal model to be effective. When the discrete time step is much smaller than the cycle time, additional linear constraints are needed to ensure that the split parameters remain the same in a full cycle. Such constraints are articulated in the set $\mathcal{U}_{1}$, and can be handled easily by our formulation.
} 
1. The problem of finding the best responsive signal strategy reduces to finding the best coefficients $A$ and $b$, which is done in an off-line optimization procedure. The on-line implementation involves only linear operation and projection as shown in (2), and can be easily performed in real time.

2. The choice of $T$ (scope of past memory) is quite flexible, allowing the decision-making process to take into account various levels of past information. Moreover, if for some incoming link the flow is not observed (e.g. a sensor is not installed), the corresponding entries in the matrix $A$ can be set to be zero.

3. The underlying feasible set for the controls, $\mathcal{U}_{1}$ or $\mathcal{U}_{2}$, can be user-defined to accommodate various realistic and practical considerations.

4. As we demonstrate subsequently, the program (3) can be formulated as a mixed integer linear program, which can be solved fairly efficiently with commercial solvers, provided that (i) the performance measure $\Phi$ is linear in all its arguments (which involves the objective function and the underlying traffic dynamics, and will be proven in Section 3); and (ii) the feasible control sets $\mathcal{U}_{1}$ or $\mathcal{U}_{2}$ satisfy certain conditions to be articulated below.

The next two propositions provide some precise conditions for the projections seen in Eqn (2) to be representable as linear constraints. These properties are important for the mixed integer linear program presented in Section 3, but can be skipped for the heuristic solution procedure presented in Section 4.

Proposition 2. (Linear representation of $\left.\mathcal{P}_{\mathcal{U}_{1}}[\cdot]\right)$ Let $\mathcal{U}_{1} \subset \mathbb{R}^{n}$ be nonempty. Assume that the feasible control set $\mathcal{U}_{1}$ can be expressed using linear constraints, i.e.,

$$
\mathcal{U}_{1}=\left\{z \in \mathbb{R}^{n}: \mathcal{A}_{1} z+c_{1}=0, \quad \mathcal{A}_{2} z+c_{2} \leq 0\right\}
$$

for appropriate matrices $\mathcal{A}_{1} \in \mathbb{R}^{m_{1} \times n}, \mathcal{A}_{2} \in \mathbb{R}^{m_{2} \times n}$ and vectors $c_{1} \in \mathbb{R}^{m_{1}}, c_{2} \in \mathbb{R}^{m_{2}}$. Then $\eta=\mathcal{P}_{\mathcal{U}_{1}}[\mu]$ is equivalent to a set of linear constraints with binary variables.

Proof. Recall from the definition of Euclidean projection that $\mathcal{P}_{\mathcal{U}_{1}}[\mu]=\operatorname{argmin}_{y \in \mathcal{U}_{1}}\left\{\frac{1}{2}\|y-\mu\|_{2}^{2}\right\}$, which reduces to a strongly convex quadratic program with linear constraints. The KKT conditions, which are sufficient for global optimality, read

$$
\eta-\mu+\mathcal{A}_{1}^{\top} \gamma_{1}+\mathcal{A}_{2}^{\top} \gamma_{2}=0, \quad 0 \leq \gamma_{2} \perp-\mathcal{A}_{2} \mu-c_{2} \geq 0, \quad \gamma_{1} \in \mathbb{R}^{m_{1}}, \gamma_{2} \in \mathbb{R}_{+}^{m_{2}}
$$

which are equivalent to the following linear constraints:

$$
\eta-\mu+\mathcal{A}_{1}^{\top} \gamma_{1}+\mathcal{A}_{2}^{\top} \gamma_{2}=0, \quad 0 \leq \gamma_{2} \leq \mathcal{M} \xi, \quad 0 \leq-\mathcal{A}_{2} \mu-c_{2} \leq \mathcal{M}(1-\xi), \quad \xi \in\{0,1\}^{m_{2}}, \gamma_{1} \in \mathbb{R}^{m_{1}}, \gamma_{2} \in \mathbb{R}_{+}^{m_{2}}
$$

where $\mathcal{M}$ is a large positive number. Thus $\eta=\mathcal{P}_{\mathcal{U}_{1}}[\mu]$ if and only if $\eta$ satisfies the linear constraints (6) plus another linear constraint $\mathcal{A}_{1} \mu+c_{1}=0$, which is stipulated by (4).

We now turn to the on-and-off signal model. Since $\mathcal{U}_{2}$ is non-convex due to the binary variables, the Euclidean projection requires solving a nonconvex program. Nevertheless, in a very special case where $\mathcal{U}_{2}=\{0,1\}^{\mathbb{\mathbb { L } _ { s }} \mid \times N}$, the projection can be represented as linear constraints.

Proposition 3. (Linear representation of $\left.\mathcal{P}_{\mathcal{U}_{2}}[\cdot]\right)$ Assume that $\mathcal{U}_{2}=\{0,1\}^{\left|\mathbb{I}_{s}\right| \times N}$, then $u \in \mathcal{P}_{\mathcal{U}_{2}}(\mu)$ if $u$ satisfies a set of linear constraints.

Proof. Under the hypothesis, the Euclidean projection problem $\min _{y \in \mathcal{U}_{2}}\left\{\frac{1}{2}\|y-\mu\|_{2}^{2}\right\}$ can be decomposed into mutually independent sub-problems $\min _{y_{i}^{t} \in\{0,1\}} \frac{1}{2}\left\|y_{i}^{t}-\mu_{i}^{t}\right\|_{2}^{2}$ for all $i \in \mathbb{L}_{s}$ and $1 \leq t \leq N$. Thus $u \in \mathcal{P}_{\mathcal{U}_{2}}(\mu)$ if it satisfies

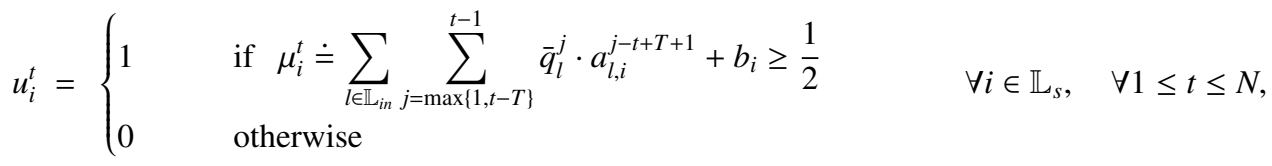

which can be easily written as a set of linear constraints with binary variables. 
If the particular forms of $\mathcal{U}_{1}$ and $\mathcal{U}_{2}$ satisfy the conditions stated in Proposition 2 and Proposition 3 , then the program (3) may be formulated as MILPs. The condition posed on $\mathcal{U}_{1}$ for the continuum signal controls is quite general and can accommodate a range of realistic situations; the condition on $\mathcal{U}_{2}$ for the on-and-off controls applies to situations where only one signal light at each intersection is freely controllable, say at a junction with two conflicting vehicle movements, and any sequence of binary control is feasible. Although the condition for $\mathcal{U}_{2}$ seems quite restrictive, and an MILP formulation is hardly attainable for real-world applications involving the on-and-off signal controls, we may still solve problem (3) using metaheuristic search methods. The metaheuristic approach will be detailed in Section 4.

\subsection{Stochastic Extension of the LDR}

The formulation (3) is most effective if the incoming traffic flow $\boldsymbol{q}_{\text {in }}$ is known exactly . In reality, however, there are naturally occurring variations in the (day-to-day) traffic flows, which may render the deterministic LDR suboptimal. A traditional approach to handle these uncertainties is stochastic programming, which relies on exact knowledge of the underlying distribution of the stochastic variables. However, in a real-world setting one can only observe ambiguous information on the distribution with finite samples. The inexact nature of our knowledge of the underlying distribution should be handled explicitly and in a robust way to ensure a sound performance of the resulting signal decisions. This is precisely the aim of the proposed distributionally robust optimization approach, which will be detailed in this subsection.

Assume that the network incoming flow vector, $\boldsymbol{q}_{i n}=\left(\bar{q}_{i}^{t}: 1 \leq t \leq N, i \in \mathbb{L}_{i n}\right)$, is a random vector following some arbitrary distribution, denoted $\mathbb{D}^{*}$. Let $\mathbb{D}_{i}^{t, *}$ be the marginal distribution of each component $\bar{q}_{i}^{t}$ for $i \in \mathbb{L}_{i n}, 1 \leq t \leq N$. None of these distributions are known a priori. We then let $\mathcal{S}=\left\{\boldsymbol{q}_{i n}^{(1)}, \boldsymbol{q}_{i n}^{(2)}, \ldots, \boldsymbol{q}_{i n}^{(K)}\right\}$ be a set of $K$ samples drawn i.i.d. from the distribution $\mathbb{D}^{*}$, which can be obtained by sampling the historical traffic flow data. Since the true distribution is unknown but can be estimated from these finite samples, there are inherent uncertainties associated with such estimations. In order to handle these uncertainties in a way that guarantees the sound performance of the optimized signals even in the least favorable situation, we propose the following distributionally robust optimization (DRO) formulation:

$$
\min _{A, b} \max _{\mathbb{D} \in \mathbb{Q}} \mathbb{E}_{\mathbb{D}} \Phi\left(\boldsymbol{q}_{\text {in }}, U\left(A, b ; \boldsymbol{q}_{\text {in }}\right)\right)
$$

subject to the traffic flow dynamics. ${ }^{3}$ Here, $\mathbb{Q}$ is the set of candidate distributions that are used to approximate the true distribution. Formulation (8) seeks to minimize, over all linear coefficients $A$ and $b$, the average (as indicated by the expectation $\mathbb{E}_{\mathbb{D}}$ ) network performance measure, in the worst-case distribution among $\mathbb{Q}$ (as indicated by $\max _{\mathbb{D} \in \mathbb{Q}}$ ). Formulation (8) is a stochastic extension of (3), and addresses the ambiguity in the distribution in a fashion similar to robust optimization. This formulation is more general than stochastic programming as in the latter case the set $\mathbb{Q}$ contains only one distribution. According to Goh and $\operatorname{Sim}(2010)$, the choice of $\mathbb{Q}$ can be fine-tuned to strike a balance between the conservatism of robust optimization and the specification of stochastic programming. The determination of $\mathbb{Q}$ is crucial to the model's performance in actual applications, and will be performed in the next subsection through a data-driven approach.

\subsection{Data-driven calibration of the uncertainty set}

We employ the Kolmogorov-Smirnov (KS) test (Massey, 1951) to construct and calibrate the candidate distribution set $\mathbb{Q}$ with given historical traffic flow data. The KS goodness-of-fit test is a popular method to test if a distribution well captures a finite set of sampled data points. Following Bertsimas et al. (2013), we incorporate the KS test-based approach to construct the uncertainty set from sampled data. The resulting $\mathbb{Q}$ contains the true distribution with a tunable probability guarantee.

\footnotetext{
${ }^{3}$ The traffic flow component of this formulation can be specified either using analytical models such as the LWR model detailed in Section 3, or through microsimulation implemented in standard software. The latter approach can be incorporated in the heuristic method discussed in Section 4.
} 
For each $i \in \mathbb{L}_{i n}, 1 \leq t \leq N$, and for a candidate marginal probability distribution $\mathbb{D}_{i}^{t}$, the KS goodness-of-fit is based on the following hypothesis testing:

$$
H_{0}: \mathbb{D}_{i}^{t}=\mathbb{D}_{i}^{t, *} \quad H_{A}: \mathbb{D}_{i}^{t} \neq \mathbb{D}_{i}^{t, *}
$$

where $\mathbb{D}_{i}^{t, *}$ is the true marginal distribution for $\bar{q}_{i}^{t}$. We let $\mathcal{S}_{i}^{t} \doteq\left\{\bar{q}_{i}^{t,(1)}, \bar{q}_{i}^{t,(2)}, \ldots, q_{i}^{t,(K)}\right\}$ be a sample set with respect to the marginal distribution $\mathbb{D}_{i}^{t, *}$, and arranged in an ascending order, i.e. $\bar{q}_{i}^{t,(1)} \leq \bar{q}_{i}^{t,(2)}, \ldots, \leq \bar{q}_{i}^{t,(K)}$. The null hypothesis $H_{0}$ is rejected at significance level $\alpha$ if

$$
\max _{j=1, \ldots, K} \max \left(\frac{j}{K}-\mathbb{P}_{i}^{t}\left(\bar{q}_{i}^{t,(j)}\right), \mathbb{P}_{i}^{t}\left(\bar{q}_{i}^{t,(j)}\right)-\frac{j-1}{K}\right)>\Theta(\alpha, K)
$$

where $K$ is the sample size, $\mathbb{P}_{i}^{t}(\cdot)$ denotes the cumulative distribution function $(\mathrm{CDF})$ of $\mathbb{D}_{i}^{t}$. In prose, the KS test rejects the null hypothesis if the value $\mathbb{P}_{i}^{t}\left(\bar{q}_{i}^{t,(j)}\right)$ is smaller or larger than certain thresholds jointly determined by the sample and a prescribed quantile of a certain distribution, denoted $\Theta(\alpha, K)$. More specifically, $\Theta(\alpha, K)$ is the $1-\alpha$ quantile of a proper null-distribution; see Stephens (1974) and Thas (2010) for more details. Following these studies, we set $\Theta(\alpha, K)=\frac{C_{\alpha}}{\sqrt{K}}$, where $C_{\alpha}$ is the solution of the equation $1-\alpha=\frac{\sqrt{2 \pi}}{x} \sum_{k=1}^{C_{M}} \exp \left(-(2 k-1)^{2} \pi^{2} /\left(8 x^{2}\right)\right)$ with unknown $x$, and $C_{M}$ is a sufficiently large integer. As a result, the confidence region, according to Bertsimas et al. (2013), is given as

$$
\mathbb{Q}_{i}^{t} \doteq\left\{\mathbb{D}_{i}^{t} \in \mathcal{B}\left[L_{i}, U_{i}\right]: \mathbb{P}_{i}^{t}\left(\bar{q}_{i}^{t,(j)}\right) \geq \frac{j}{K}-\Theta(\alpha, K), \mathbb{P}_{i}^{t}\left(\bar{q}_{i}^{t,(j)}\right) \leq \frac{j-1}{K}+\Theta(\alpha, K), \quad \forall 1 \leq j \leq K\right\}
$$

where $L_{i}$ and $U_{i}$ are respectively some fixed lower and upper bounds on the sampled inflows, and they usually depend on the link $i \in \mathbb{L}_{i n} . \mathcal{B}\left[L_{i}, U_{i}\right]$ denotes the set of Borel measures on $\left[L_{i}, U_{i}\right]$. In prose, $\mathbb{Q}_{i}^{t}$ consists of candidate marginal distributions with which the null hypothesis will not be rejected at significance level $\alpha$. The choice of $\alpha$ impacts the trade-off between robustness and optimality. A smaller $\alpha$ results in a larger uncertainty set (confidence region), and thus the solution of the DRO problem (8) becomes more robust against uncertainty. In the meantime, however, a larger uncertainty set renders the DRO more conservative, leading to worsened average performance.

We will next re-write (11) in terms of probability density functions $(\mathrm{PDFs})$, denoted by $\mathbb{F}_{i}^{t}(\cdot)$. Given that there are only finite number of samples, we invoke a piecewise constant approximation of $\mathbb{F}_{i}^{t}(\cdot)$, which is illustrated in Figure 1. Accordingly, the $\mathrm{CDF} \mathbb{P}_{i}^{t}(\cdot)$ is approximated using the sum of areas of the rectangles. We readily derive, based on (11) and the piecewise constant approximation, the following characterization of suitable candidate distributions in terms of their PDFs.

$\tilde{\mathbb{Q}}_{i}^{t} \doteq\left\{\mathbb{D}_{i}^{t} \in \mathcal{B}\left[L_{i}, U_{i}\right]: \frac{\max \left\{0, \frac{1}{K}-2 \Theta(\alpha, K)\right\}}{\bar{q}_{i}^{t,(j)}-\bar{q}_{i}^{t,(j-1)}} \leq \mathbb{F}_{i}^{t}\left(\bar{q}_{i}^{t}\right) \leq \frac{\frac{1}{K}+2 \Theta(\alpha, K)}{\bar{q}_{i}^{t,(j)}-\bar{q}_{i}^{t,(j-1)}} \quad \bar{q}_{i}^{t} \in\left(\bar{q}_{i}^{t,(j-1)}, \bar{q}_{i}^{t,(j)}\right], \quad \forall 2 \leq j \leq K\right\}$

Notice that $\tilde{\mathbb{Q}}_{i}^{t}$ strictly contains $\mathbb{Q}_{i}^{t}$, which indicates that the former is more conservative in the robust optimization formulation.

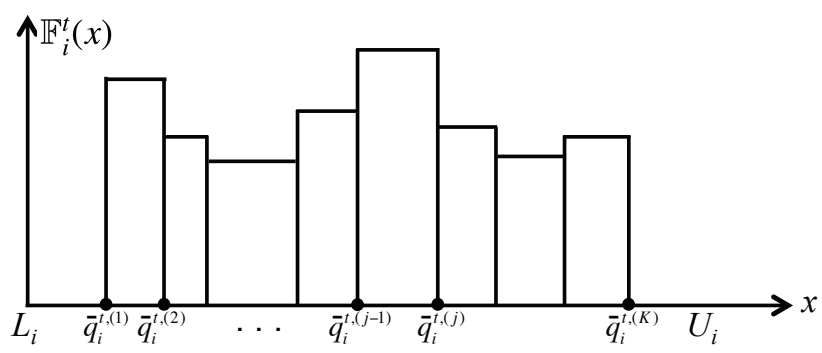

Fig. 1: Piecewise-constant approximation of the probability distribution function driven by data samples. 


\section{The mixed integer linear programming approach for the off-line computation}

This section derives the mixed integer linear program (MILP) formulation for solving the complete linear decision rule (LDR) based distributionally robust optimization (DRO) problem (8). The MILP belongs to the off-line stage of the proposed on-line signal control. The MILP employs the Lighthill-Whitham-Richards (LWR) model to describe network dynamics, and a novel reformulation of the "minimax" problem (8) based on dual variables. The resulting MILP can be efficiently solved with commercial solvers and is tested in our numerical study in Section 5.

\subsection{Formulation of traffic network dynamics}

This section presents discrete-time dynamics of the traffic flows on signalized networks based on the LWR model. Both the on-and-off and the continuum signal controls will be incorporated. Consider a fixed planning horizon $[0, \mathcal{T}]$, uniformly partitioned into $N$ time intervals labeled by $t=1, \ldots, N$. The time step size is denoted by $\delta t$. Throughout this paper, we use superscript $t$ to indicate the discrete time step. The fundamental diagrams of all relevant links in the network are assumed to be triangular expressed as:

$$
f_{i}(\rho)=\left\{\begin{array}{ll}
v_{i} \rho & \text { if } \rho \in\left[0, \rho_{i}^{c}\right] \\
-w_{i}\left(\rho-\rho_{i}^{j a m}\right) & \text { if } \rho \in\left(\rho_{i}^{c}, \rho_{i}^{j a m}\right]
\end{array} \quad \forall i \in \mathbb{L}\right.
$$

where the subscript $i$ indicates link ID, and $\mathbb{L}$ denotes the set of links. $v_{i}$ and $w_{i}$ denote the forward and backward kinematic wave speeds, respectively; $\rho_{i}^{c}$ denotes the critical density at which the flow is maximized; and $\rho_{i}^{j a m}$ denotes the jam density. Furthermore, we let $C_{i}$ be the flow capacity and $L_{i}$ be the length of the link.

\subsubsection{Link dynamics}

The propagation of kinematic waves and congestion within and between links is captured by a link-based approach (Yerman et al., 2005; Han et al., 2012). We define $\Delta_{i}^{f} \doteq\left\lceil\frac{L_{i}}{v_{i} \delta t}\right\rceil, \Delta_{i}^{b} \doteq\left\lceil\frac{L_{i}}{w_{i} \delta t}\right\rceil$, where $\lceil x\rceil$ rounds a real number $x$ to the nearest integer from above. The following notations are introduced, where the superscript $t$ always denotes the discrete time step; and we use "bar" ("hat") to indicate quantities associated with the entrance (exit) of a link.

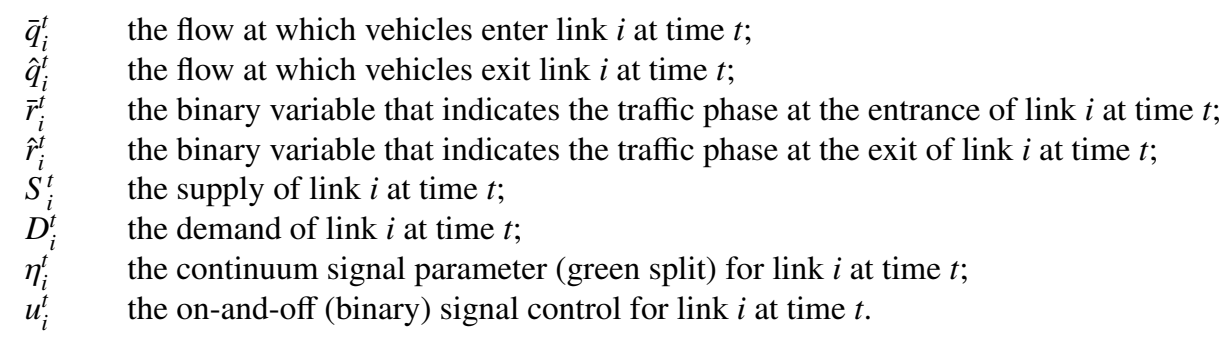

Here, the binary variables $\bar{r}_{i}^{t}$ (or $\hat{r}_{i}^{t}$ ) equal zero if traffic is in the free-flow phase at the entrance (or exit) of the link, and equal one if traffic is in the congested phase at the entrance (or exit). The two phases correspond to the left and right branches of the triangular fundamental diagram, respectively. As we subsequently explain, these binary variables can be determined using the variational approach. The demand $D_{i}^{t}$ and supply $S_{i}^{t}$ (both interpreted as flow) express respectively the maximum sending and receiving capacity of the link, and are consistent with those defined in the literature (Lebacque and Khoshyaran, 1999). According to the variational theory (Newell, 1993; Yerman et al., 2005), the aforementioned binary variables can be determined as follows. 


$$
\begin{gathered}
\bar{r}_{i}^{t}= \begin{cases}1 & \text { if } \quad \delta t \sum_{l=1}^{t} \bar{q}_{i}^{l}=\delta t \sum_{l=1}^{t-\Delta_{i}^{b}} \hat{q}_{i}^{l}+\rho_{i}^{j a m} L_{i} \\
0 & \text { if } \quad \delta t \sum_{l=1}^{t} \bar{q}_{i}^{l}<\delta t \sum_{l=1}^{t-\Delta_{i}^{b}} \hat{q}_{i}^{l}+\rho_{i}^{j a m} L_{i}\end{cases} \\
\Delta_{i}^{b}+1 \leq t \leq N
\end{gathered}
$$

$$
\begin{gathered}
\hat{r}_{i}^{t}= \begin{cases}0 & \text { if } \delta t \sum_{l=1}^{t-\Delta_{i}^{f}} \bar{q}_{i}^{l}=\delta t \sum_{l=1}^{t} \hat{q}_{i}^{l} \\
1 & \text { if } \delta t \sum_{l=1}^{t-\Delta_{i}^{f}} \bar{q}_{i}^{l}>\delta t \sum_{l=1}^{t} \hat{q}_{i}^{l}\end{cases} \\
\Delta_{i}^{f}+1 \leq t \leq N
\end{gathered}
$$

Under the assumption that the network is initially empty, we must have $\bar{r}_{i}^{t}=0$ for $t \leq \Delta_{i}^{b}$ and $\hat{r}_{i}^{t}=0$ for $t \leq \Delta_{i}^{f}$. In (14), we have expressed cumulative vehicle counts in terms of discrete-time integrals of the flow variables. The reader is referred to Han et al. (2014) for a detailed derivation of the above identities using the variational theory. Eqn (14) leads to the following mixed integer inequality constraints.

$$
\begin{aligned}
& \left\{\begin{array}{l}
\delta t \sum_{l=1}^{t} \bar{q}_{i}^{l}+\mathcal{M}\left(1-\bar{r}_{i}^{t}\right) \geq \delta t \sum_{l=1}^{t-\Delta_{i}^{b}} \hat{q}_{i}^{l}+\rho_{i}^{j a m} L_{i}-\varepsilon \\
\delta t \sum_{l=1}^{t} \bar{q}_{i}^{l}-\mathcal{M} \bar{r}_{i}^{t} \leq \delta t \sum_{l=1}^{t-\Delta_{i}^{b}} \hat{q}_{i}^{l}+\rho_{i}^{j a m} L_{i}-\varepsilon
\end{array}\right. \\
& \Delta^{b}+1 \leq t \leq N
\end{aligned}
$$$$
\begin{aligned}
& \left\{\begin{array}{l}
\delta t \sum_{l=1}^{t-\Delta_{i}^{f}} \bar{q}_{i}^{l}-\mathcal{M} \hat{r}_{i}^{t} \leq \delta t \sum_{l=1}^{t} \hat{q}_{i}^{l}+\varepsilon \\
\delta t \sum_{l=1}^{t-\Delta_{i}^{f}} \bar{q}_{i}^{l}-\mathcal{M}\left(\hat{r}_{i}^{t}-1\right) \geq \delta t \sum_{l=1}^{t} \hat{q}_{i}^{l}+\varepsilon
\end{array}\right. \\
& \Delta_{i}^{f}+1 \leq t \leq N
\end{aligned}
$$

where $\mathcal{M}>0$ is a very large number, and $\varepsilon>0$ is a very small number serving as a cutoff threshold. Once the traffic phases are determined, the demand (supply) of the link is determined via the binary variables $\hat{r}_{i}^{t}\left(\bar{r}_{i}^{t}\right)$ as:

$$
D_{i}^{t}=\left\{\begin{array}{ll}
C_{i} & \text { if } \hat{r}_{i}^{t}=1 \\
\bar{q}_{i}^{t-\Delta_{i}^{f}} & \text { if } \hat{r}_{i}^{t}=0
\end{array} \quad S_{i}^{t}= \begin{cases}\hat{q}^{t-\Delta_{i}^{b}} & \text { if } \bar{r}_{i}^{t}=1 \\
C_{i} & \text { if } \bar{r}_{i}^{t}=0\end{cases}\right.
$$

where $C_{i}$ denotes the link flow capacity. Similarly, these logical expressions admit linear inequality representations:

$$
\left\{\begin{array} { l } 
{ C _ { i } + \mathcal { M } ( \hat { r } _ { i } ^ { t } - 1 ) \leq D _ { i } ^ { t } \leq C _ { i } } \\
{ \overline { q } _ { i } ^ { t - \Delta _ { i } ^ { f } } - \mathcal { M } \hat { r } _ { i } ^ { t } \leq D _ { i } ^ { t } \leq \overline { q } _ { i } ^ { t - \Delta _ { i } ^ { f } } + \mathcal { M } \hat { r } _ { i } ^ { t } }
\end{array} \quad \left\{\begin{array}{l}
C_{i}-\mathcal{M} \bar{r}_{i}^{t} \leq S_{i}^{t} \leq C_{i} \\
\hat{q}_{i}^{t-\Delta_{i}^{b}}-\mathcal{M}\left(1-\bar{r}_{i}^{t}\right) \leq S_{i}^{t} \leq \hat{q}_{i}^{t-\Delta_{i}^{b}}+\mathcal{M}\left(1-\bar{r}_{i}^{t}\right)
\end{array}\right.\right.
$$

\subsubsection{Junction dynamics}

We consider a signalized junction $J$ with the set of incoming links denoted by $\mathcal{I}^{J}$ and the set of outgoing links denoted by $O^{J}$. For every $i \in \mathcal{I}^{J}$, we let $\eta_{i}^{t} \in(0,1)$ be the green split allocated to this link. The continuum signal model reads

$$
\hat{q}_{i}^{t}=\min \left\{D_{i}^{t}, \eta_{i}^{t} \cdot \min \left\{C_{i}, \min _{j \in O^{J}}\left\{\frac{S_{j}^{t}}{\alpha_{i, j}^{t}}\right\}\right\}\right\} \quad \forall i \in \mathcal{I}^{J}, \quad \forall 1 \leq t \leq N \quad \text { (continuum signal model) }
$$

where the turning ratio $\alpha_{i, j}^{t}$ represents, within every unit of flow exiting link $i$ at time $t$, its proportion that advances into link $j$. Throughout this paper these turning ratios are known a priori, which can be estimated from turn-by-turn vehicle counts. The quantity $\min \left\{C_{i}, \min _{j \in O^{J}}\left\{S_{j}^{t} / \alpha_{i, j}^{t}\right\}\right\}$ is termed effective supply for link $i$ to refer to the downstream capacity available to link $i$; and it is allocated to link $i$ according to the signal split parameter $\eta_{i}^{t}$.

Alternatively, one could consider the more traditional on-and-off signal control by defining the binary variable $u_{i}^{t} \in\{0,1\}$, which represents the red/green phases of a signal light for road $i$ at time $t$. The on-and-off model reads

$$
\hat{q}_{i}^{t}=\min \left\{D_{i}^{t}, u_{i}^{t} \cdot \min \left\{C_{i}, \min _{j \in O^{J}}\left\{\frac{S_{j}^{t}}{\alpha_{i, j}^{t}}\right\}\right\}\right\} \quad \forall i \in \mathcal{I}^{J}, \quad \forall 1 \leq t \leq N \quad \text { (on-and-off signal model) }
$$


Remark 1. Han et al. (2014) show that the continuum signalized junction model (18) is a good approximation of the on-and-off signal model (19) provided that spillback does not occur at these intersections; otherwise the continuum approximation may introduce significant errors. Thus, to ensure the approximation efficacy of the continuum model (18), and also to avoid severe congestion, one could prevent spillback from happening by stipulating that $\bar{r}_{i}^{t}=0$ for all $t$, which, according to (15), translates into a set of linear constraints:

$$
\delta t \sum_{l=1}^{t} \bar{q}_{i}^{l} \leq \delta t \sum_{l=1}^{t-\Delta_{i}^{b}} \hat{q}_{i}^{l}+\rho_{i}^{j a m} L_{i}-\varepsilon \quad \forall 1 \leq t \leq N, \quad i \in \mathbb{L}
$$

Both the continuum signal model (18) and the on-and-off signal model (19) can be expressed as linear constraints with the aid of additional binary variables and the "big-M" method to handle the "min" operator. This is a standard techinique and will not be detailed here due to space limitation.

\subsubsection{Objective function}

In this paper we employ the following form of the linear objective:

$$
\min \sum_{t=1}^{N} \sum_{i \in \mathbb{L}_{\text {out }}} \frac{\delta t}{t+1} \cdot \hat{q}_{i}^{t}
$$

where $\mathbb{L}_{\text {out }}$ denotes the set of outgoing links of the network. Such an objective is a weighted sum of the network throughput at each time instance. (21) tends to minimize queuing in the network and maximize the network throughput at every point in time. Note that other types of linear objectives proposed in the literature can be equally applied without affecting the problem formulation.

\subsection{MILP formulation of the LDR-DRO problem}

This section presents the complete MILP formulation for solving the LDR-DRO problem (8) (the off-line problem of the responsive signal control), by combining discussions from Section 2 and Section 3.1 with a dual reformulation of the minimax problem and a finite sample approximation approach. Special attention are given to the practical concerns regarding computational tractability and convenience in numerical implementations.

For the MILP formulation, we assume that all the entries, $\bar{q}_{i}^{t}$ for $i \in \mathbb{L}_{i n}, 1 \leq t \leq N$, are mutually independent (this assumption will be dropped in Section 4 for the metaheuristic approach). Therefore, in view of (12), the set of candidate distributions for the entire random vector $\boldsymbol{q}_{\text {in }}$ is immediately given as:

$$
\begin{aligned}
& \mathbb{Q} \doteq\left\{\mathbb{D} \in \mathcal{B}\left(\prod_{i \in \mathbb{L}_{\text {in }}}\left[L_{i}, U_{i}\right]^{N}\right):\left(\max \left\{0, \frac{1}{K}-2 \Theta(\alpha, K)\right\}\right)^{N \times\left|\mathbb{L}_{i n}\right|} \prod_{t=1}^{N} \prod_{i \in \mathbb{L}_{\text {in }}}\left(\sum_{j=1}^{K} \frac{\mathbb{I}\left(\bar{q}_{i}^{t} \in\left(\bar{q}_{i}^{t,(j-1)}, \bar{q}_{i}^{t,(j)}\right]\right)}{\bar{q}_{i}^{t,(j)}-\bar{q}_{i}^{t,(j-1)}}\right)\right\} \\
& \left.\leq \mathbb{F}\left(\boldsymbol{q}_{i n}\right) \leq\left(\frac{1}{K}+2 \Theta(\alpha, K)\right)^{\mathbb{L} \mathbb{L}_{i n} \mid \cdot N} \prod_{i=1}^{N} \prod_{l \in \mathbb{L}}\left(\sum_{j=1}^{K} \frac{\mathbb{I}\left(\bar{q}_{i}^{t} \in\left(\bar{q}_{i}^{t,(j-1)}, \bar{q}_{i}^{t,(j)}\right]\right)}{\bar{q}_{i}^{t,(j)}-\bar{q}_{i}^{t,(j-1)}}\right), \quad \forall \boldsymbol{q}_{i n} \in \prod_{i \in \mathbb{L}_{\text {in }}}\left[L_{i}, U_{i}\right]^{N}\right\}
\end{aligned}
$$

where the index function $\mathbb{I}(y)$ equals one if $y$ is true and zero if $y$ is false, $\mathbb{F}(\cdot)$ is the PDF of $\mathbb{D}$. The set $\mathbb{Q}$ contains candidate distributions for the distributionally robust optimization problem. Here, for notation convenience we call the lower and upper bounds in (22) $L_{\mathbb{D}}\left(\boldsymbol{q}_{i n}\right)$ and $U_{\mathbb{D}}\left(\boldsymbol{q}_{i n}\right)$, respectively.

To further simply the notations, we re-write (8) using the following compact and more general notations:

$$
\min _{x \in X} \max _{\mathbb{D} \in \mathbb{Q}} \mathbb{E}_{\mathbb{D}}[f(x, \boldsymbol{q})]
$$

where $\boldsymbol{q}=\left(q_{i}: 1 \leq i \leq M\right)$ is a random vector whose probability distribution is approximated by members of the set $\mathbb{Q}$; and it is instantiated by the random incoming flow vector $\boldsymbol{q}_{i n} . f(x, \boldsymbol{q})$ is instantiated by the performance measure $\Phi\left(\boldsymbol{q}_{\text {in }}, U\left(A, b ; \boldsymbol{q}_{i n}\right)\right)$ with $x$ corresponding to the LDR coefficients $(A, b)$. The set $\mathbb{Q}$, originally given by (22), is simplified to be

$$
\mathbb{Q} \doteq\left\{\mathbb{D} \in \mathcal{B}(\mathbb{S}): L_{\mathbb{D}}(\boldsymbol{q}) \leq \mathbb{F}(\boldsymbol{q}) \leq U_{\mathbb{D}}(\boldsymbol{q}), \forall \boldsymbol{q} \in \mathbb{S}\right\}
$$


where $\mathbb{S} \doteq \prod_{i \in \mathbb{L}_{\text {in }}}\left[L_{i}, U_{i}\right]^{N} \subset \mathbb{R}^{M}$ denotes the intersection of the support of $\boldsymbol{q}$ and the domain of $f(x, \cdot)$, and $M=$ $\left|\mathbb{L}_{\text {in }}\right| \times N$. Indeed, (23)-(24) form an abstract and more general problem than (8) and (22). All of our analyses subsequently presented focus on (23)-(24), and they immediately apply to the original LDR-DRO problem.

Problem (23) is the same as the following:

$$
\min _{x \in X} \max _{\mathbb{D} \in \mathbb{Q}}\left[\int_{\mathbb{S}} f(x, \boldsymbol{q}) \mathbb{F}(\boldsymbol{q}) d \boldsymbol{q}\right]
$$

Notice that problem (25) is a minimax (bi-level) problem, which is difficult to solve exactly. In the following, we seek to derive a single-level reformulation that serves to approximate (25). This new formulation can be directly solved by commercial solvers with significantly less computational overhead than the minimax problem. Derivation of the proposed alternative formulation involves two steps: (1) a dual reformulation, which reduces the minimax formulation to a single-level minimization problem; (2) a finite sampling approach to approximate the integrals, for which theoretical error bounds are available.

\subsubsection{The dual reformulation}

We consider the inner problem of the minimax problem (notice the negative sign in the objective):

$$
Z_{1} \doteq \min _{\mathbb{D} \in \mathbb{Q}} \int_{\mathbb{S}}-f(x, \boldsymbol{q}) \mathbb{F}(\boldsymbol{q}) d \boldsymbol{q} \quad \text { subject to } \quad \int_{\mathbb{S}} \mathbb{F}(\boldsymbol{q}) d \boldsymbol{q}=1, \quad \mathbb{F}(\boldsymbol{q}) \geq 0, \quad L_{\mathbb{D}}(\boldsymbol{q}) \leq \mathbb{F}(\boldsymbol{q}) \leq U_{\mathbb{D}}(\boldsymbol{q})
$$

The Lagrangian dual problem is formulated as maximizing the following functional:

$$
\begin{aligned}
\mathcal{L}\left(\beta_{1}, \beta_{2}, \lambda, v\right)= & \min _{\mathbb{D}}-\int_{\mathbb{S}} f(x, \boldsymbol{q}) \mathbb{F}(\boldsymbol{q}) d \boldsymbol{q}+\lambda\left(\int_{\mathbb{S}} \mathbb{F}(\boldsymbol{q}) d \boldsymbol{q}-1\right)-\int_{\mathbb{S}} v(\boldsymbol{q}) \mathbb{F}(\boldsymbol{q}) d \boldsymbol{q} \\
& \left.+\int_{\mathbb{S}}\left[\beta_{1}(\boldsymbol{q})\left(L_{\mathbb{D}}(\boldsymbol{q})-\mathbb{F}(\boldsymbol{q})\right)+\beta_{2}(\boldsymbol{q})\left(\mathbb{F}(\boldsymbol{q})-U_{\mathbb{D}}(\boldsymbol{q})\right)\right)\right] d \boldsymbol{q} \\
= & \min _{\mathbb{D}} \int_{\mathbb{S}}\left[-f(x, \boldsymbol{q})+\lambda-v(\boldsymbol{q})-\beta_{1}(\boldsymbol{q})+\beta_{2}(\boldsymbol{q})\right] \mathbb{F}(\boldsymbol{q}) d \boldsymbol{q}-\lambda \\
& +\int_{\mathbb{S}}\left[\beta_{1}(\boldsymbol{q}) L_{\mathbb{D}}(\boldsymbol{q})-\beta_{2}(\boldsymbol{q}) U_{\mathbb{D}}(\boldsymbol{q})\right] d \boldsymbol{q}, \quad \beta_{1}(\boldsymbol{q}) \geq 0, \beta_{2}(\boldsymbol{q}) \geq 0, v(\boldsymbol{q}) \geq 0
\end{aligned}
$$

The well-definedness of the dual problem is ensured by $-f(x, \boldsymbol{q})+\lambda-\beta_{1}(\boldsymbol{q})+\beta_{2}(\boldsymbol{q}) \geq 0$. With this constraint, one trivial minimizer of (28) is $\mathbb{F}(\boldsymbol{q}) \equiv 0, \forall \boldsymbol{q} \in \mathbb{S}$. Thus, for a given $x \in X,(28)$ is reduced to the following

$$
\begin{aligned}
& \quad Z_{2} \doteq \max _{\beta_{1}, \beta_{2}, \lambda} \int_{\mathbb{S}}\left[\beta_{1}(\boldsymbol{q}) L_{\mathbb{D}}(\boldsymbol{q})-\beta_{2}(\boldsymbol{q}) U_{\mathbb{D}}(\boldsymbol{q})\right] d \boldsymbol{q}-\lambda \\
& \text { subject to } \quad-f(x, \boldsymbol{q})+\lambda-\beta_{1}(\boldsymbol{q})+\beta_{2}(\boldsymbol{q}) \geq 0, \forall \boldsymbol{q} \in \mathbb{S}, \quad \beta_{1}(\boldsymbol{q}) \geq 0, \beta_{2}(\boldsymbol{q}) \geq 0, \forall \boldsymbol{q} \in \mathbb{S} .
\end{aligned}
$$

It can be easily verified that the weak duality holds between the primal and the Lagrangian dual, i.e., $Z_{2} \leq Z_{1}$. This means that we can improve the objective of the original problem by minimizing the alternative formulation (29)(30). Under proper regularity conditions analogous to the constraint qualifications for finite-dimensional mathematical programs, the strong duality may hold, i.e., $Z_{1}=Z_{2}$. Such regularity conditions are discussed in Bertsimas and Popescu (2005), which will not be elaborated in this paper. Thanks to the dual formulation, we have a single-level conservative approximation of the original problem (23)-(24):

$$
\begin{array}{cl}
\min _{x \in X, \beta_{1}, \beta_{2}, \lambda} & \int_{\mathbb{S}}\left[-\beta_{1}(\boldsymbol{q}) L_{\mathbb{D}}(\boldsymbol{q})+\beta_{2}(\boldsymbol{q}) U_{\mathbb{D}}(\boldsymbol{q})\right] d \boldsymbol{q}+\lambda \\
\text { subject to } & -f(x, \boldsymbol{q})+\lambda-\beta_{1}(\boldsymbol{q})+\beta_{2}(\boldsymbol{q}) \geq 0, \forall \boldsymbol{q} \in \mathbb{S} \\
& \beta_{1}(\boldsymbol{q}) \geq 0, \beta_{2}(\boldsymbol{q}) \geq 0, \forall \boldsymbol{q} \in \mathbb{S}
\end{array}
$$




\subsubsection{Finite sample approximation and the mixed integer linear program}

The dual problem (31)-(33) cannot be directly solved due to its continuous (infinite-dimensional) objective and semi-infinite constraint (32). To resolve this, we invoke a finite sample approximation as follows. We consider a random vector $\boldsymbol{p}=\left(p_{i}: 1 \leq i \leq M\right)$ that follows a uniform distribution on $\mathbb{S}=\prod_{i=1}^{M}\left[L_{i}, U_{i}\right]$ where each component $p_{i}$ follows a uniform distribution in $\left[L_{i}, U_{i}\right]$ and is independent of each other. We conduct a random sampling of $\boldsymbol{p}$ and obtain a set of $\mathcal{K}$ i.i.d. samples $\left\{\boldsymbol{p}^{(k)}=\left(p_{i}^{(k)}: 1 \leq i \leq M\right): k=1, \ldots, \mathcal{K}\right\} \subset \mathbb{S}$. Then, we use this sample set to construct the following finite dimensional problem to approximate (31)-(33):

$$
\begin{aligned}
& \min _{\beta_{1, k}, \beta_{2, k}, \lambda} \frac{1}{\mathcal{K}} \sum_{k=1}^{\mathcal{K}}\left[-\beta_{1, k} L_{\mathbb{D}}\left(\boldsymbol{p}^{(k)}\right)+\beta_{2, k} U_{\mathbb{D}}\left(\boldsymbol{p}^{(k)}\right)\right] \prod_{i=1}^{M}\left(U_{i}-L_{i}\right)+\lambda \\
& \text { subject to } \quad-f\left(x, \boldsymbol{p}^{(k)}\right)+\lambda-\beta_{1, k}+\beta_{2, k} \geq 0, \quad \forall 1 \leq k \leq \mathcal{K} \\
& \beta_{1, k} \geq 0, \beta_{2, k} \geq 0, \quad \forall 1 \leq k \leq \mathcal{K} \quad
\end{aligned}
$$

The efficacy of the proposed finite sample approach (34)-(36) is guaranteed theoretically: two theorems are provided in Appendix A to rigorously assess the approximation accuracy of the proposed finite sample scheme. Problem (34)(36) gives rise to the MILP formulation of the original problem provided that the function $f\left(x, \boldsymbol{p}^{(k)}\right)$ is linear in $x$, which is indeed the case due to the linear representation of the traffic dynamics and the objective function in Section 3.1, and the linear representation of the Euclidean projection discussed in Section 2.1. More specifically, the complete MILP is formed by Eqns (34)-(36), in which $f\left(x, \boldsymbol{p}^{(k)}\right)$, instantiated by $\Phi\left(\boldsymbol{q}^{(k)}, U\left(A, b ; \boldsymbol{q}^{(k)}\right)\right)$, is expressed in terms of (6) (or (7)), (15), (17), (18) (or (19)), (20), and (21).

\section{Metaheuristic approach for the off-line computation}

This section proposes, for the LDR-DRO problem, a metaheuristic search method that complements the MILP approach in several important ways.

1. It can be applied to large-scale and more realistic signal control problems. The MILP may consume substantial amount of computational resource when the problem size scales up, while the metaheuristics provides a more flexible trade-off between computational overhead and solution quality and often yields good solutions with reasonable computational cost.

2. In contrast to the MILP approach, the metaheuristic does not rely on the assumption that the components of the incoming flow vector are mutually independent. This is a desirable generalization as incoming flows from different links at different times are likely to be correlated in reality.

3. The incorporation of realistic requirements/constraints at signalized intersections requires solving an Euclidean projection problem onto $\mathcal{U}_{1}$ or $\mathcal{U}_{2}$, which may not admit explicit or tractable reformulations if those constraints are sufficiently sophisticated and nonlinear. This is, however, not a problem for the metaheuristic approach as it only requires zero ${ }^{\text {th }}$-order information on the objective function. Thus the Euclidean projection can be performed within a simulation procedure used to determined the objective values.

We employ the particle swarm optimization (PSO) technique (Kennedy and Eberhart, 1995; Banks et al., 2007), noting that other types of metaheuristics may be applied as well with minimum modification. The PSO requires only zero $^{\text {th }}$-order information of the objective and the constraints, which will be evaluated through a Monte Carlo approach.

\subsection{Evaluating the worst-case objective value}

Our analysis and algorithm presented subsequently do not rely on the assumption that the individual components in the network inflow vector are mutually independent. In this case the approach employed in Section 3.2 for calibrating the candidate distribution set does not apply. Instead, we consider the following formulation, which is slightly different from (23):

$$
\min _{x \in X} \max _{\mathbb{D}_{f} \in \mathbb{Q}_{f}(x)} \mathbb{E}_{\mathbb{D}_{f}}[f(x, \boldsymbol{q})]
$$


Here, $f(x, \boldsymbol{q}) \in \mathbb{R}$ is viewed as a random variable due to the stochasticity in $\boldsymbol{q}$, and is parameterized by $x$, which is instantiated by the LDR coefficients $(A, b)$. The set of candidate distributions for $f(x, \boldsymbol{q})$, which contains the true distribution, is thus dependent on $x$ as well, and is denoted by $\mathbb{Q}_{f}(x)$. We may now invoke the KS-test approach for the random variable $f(x, \boldsymbol{q})$ to construct $\mathbb{Q}_{f}(x)$ for a prescribed $x \in X$. Let $\mathcal{S}=\left\{\boldsymbol{q}^{(1)}, \boldsymbol{q}^{(2)}, \ldots, \boldsymbol{q}^{(K)}\right\}$ be the set of $K$ samples drawn i.i.d. from the true distribution. For each such $\mathcal{S}$, we can obtain a sequence of objective function values $\left\{f^{(j)}(x) \doteq f\left(x, \boldsymbol{q}^{(j)}\right)\right\}_{1 \leq j \leq K}$, arranged in an ascending order without loss of generality. This sequence can be used in the KS-test to construct the distribution for the random variable $f(x, \boldsymbol{q})$. We fix a lower bound $L_{f}$ and an upper bound $U_{f}$ of the objective value $f(x, \boldsymbol{q})$. Then, similar to the construction of (12), we can calibrate a set $\mathbb{Q}_{f}(x)$ of candidate distributions as follows. Here, $\mathbb{D}_{f} \in \mathbb{Q}_{f}(x)$, and has a corresponding PDF denoted by $\mathbb{F}_{f}(\cdot)$.

$\mathbb{Q}_{f}(x) \doteq\left\{\mathbb{D}_{f} \in \mathcal{B}\left[L_{f}, U_{f}\right]: \frac{\max \left\{0, \frac{1}{K}-2 \Theta(\alpha, K)\right\}}{f^{(j)}(x)-f^{(j-1)}(x)} \leq \mathbb{F}_{f}(y) \leq \frac{\frac{1}{K}+2 \Theta(\alpha, K)}{f^{(j)}(x)-f^{(j-1)}(x)}, \quad y \in\left(f^{(j-1)}(x), f^{(j)}(x)\right], \quad \forall 2 \leq j \leq K\right\}$

With such a characterization of the candidate distributions, we next focus on the numerical evaluation of the worst-case objective value $\max _{\mathbb{D}_{f} \in \mathbb{Q}_{f}(x)} \mathbb{E}_{\mathbb{D}_{f}}[f(x, \boldsymbol{q})]$ by piecewise-constant approximation: Fix any uniform partition $\left\{g_{i}\right\}_{1 \leq i \leq W}$ of the interval $\left[L_{f}, U_{f}\right]$ with step size denoted by $\Delta$. We let $\mathbb{F}_{f, i} \doteq \mathbb{F}_{f}\left(g_{i}\right), \forall 1 \leq i \leq W$. Then

$$
\max _{\mathbb{D}_{f} \in \mathbb{Q}_{f}(x)} \mathbb{E}_{\mathbb{D}_{f}}[f(x, \boldsymbol{q})] \approx \max _{\left(\mathbb{F}_{f, i}: 1 \leq i \leq W\right)}\left\{\sum_{i=1}^{W} g_{i} \cdot \mathbb{F}_{f, i} \cdot \Delta: \sum_{i=1}^{W} \mathbb{F}_{f, i} \cdot \Delta=1 ; \mathbb{F}_{f, i} \geq 0, L_{f, i} \leq \mathbb{F}_{f, i} \leq U_{f, i}, \forall 1 \leq i \leq W\right\}
$$

where the constants $L_{f, i}$ and $U_{f, i}$ directly come from (38):

$L_{f, i} \doteq \sum_{j=1}^{K} \frac{\max \left\{0, \frac{1}{K}-2 \Theta(\alpha, K)\right\}}{f^{(j)}(x)-f^{(j-1)}(x)} \mathbb{I}\left(g_{i} \in\left(f^{(j-1)}(x), f^{(j)}(x)\right]\right), \quad U_{f, i} \doteq \sum_{j=1}^{K} \frac{\frac{1}{K}+2 \Theta(\alpha, K)}{f^{(j)}(x)-f^{(j-1)}(x)} \mathbb{I}\left(g_{i} \in\left(f^{(j-1)}(x), f^{(j)}(x)\right]\right)$

The right hand side of (39) is viewed as a linear program, the solution of which approximates the expectation of the performance measure in the worst-case scenario. Notice that the left hand side of (39) is the objective value subject to minimization in our PSO-based optimization.

In summary, we have the following procedure for evaluating the objective function of the PSO-based optimization, with given control $x$ and sampled dataset $\mathcal{S}$. Notice that a uniform partition $\left\{g_{i}\right\}_{1 \leq i \leq W}$ of $\left[L_{f}, U_{f}\right]$ is prescribed for the following algorithm.

\section{Algorithm 1: Pseudo code for evaluating the objective function}

Input Sampled dataset $\mathcal{S}=\left\{\boldsymbol{q}^{(1)}, \ldots, \boldsymbol{q}^{(K)}\right\}$, control $x=(A, b)$,

Step 1 For each $1 \leq j \leq K$, conduct traffic simulation to calculate $f^{(j)}(x)=f\left(x, \boldsymbol{q}^{(j)}\right)=\Phi\left(\boldsymbol{q}^{(j)}, U\left(A, b ; \boldsymbol{q}^{(j)}\right)\right)$.

Step 2 Calculate $L_{f, i}$ and $U_{f, i}$ according to (40) for all $1 \leq i \leq W$.

Step 3 Evaluate the worst-case expected performance $Y$ by solving the linear program in (39). Return $Y$.

\subsection{The computational procedures based on particle swarm optimization (PSO)}

The following pseudo code of PSO for solving the complete off-line problem (23) is tailored to incorporate all of our discussion presented in this section so far. We refer the reader to Banks et al. (2007) for a general introduction and a comprehensive review of PSO. 
Algorithm 2: Pseudo code for solving the off-line problem (37)

Input Define the feasible region $X \in \mathbb{R}^{m}$ of the optimization problem. Obtain a set $\mathcal{S}$ of sampled network inflow vectors, and select a significance level $\alpha$. Fix a positive integer Pop, which denotes the population of the swarm (number of search agents).

Step 0 Set iteration counter $\tau=0$. Randomly initialize the positions $\left\{x_{n}^{\tau}\right\}_{1 \leq n \leq P o p} \subset X$ and velocities $\left\{V_{n}^{\tau}\right\}_{1 \leq n \leq P o p} \subset$ $\mathbb{R}^{m}$. Set $P_{n}^{\tau}=x_{n}^{\tau}, 1 \leq n \leq$ Pop. Let $G^{\tau} \in X$ be arbitrary. Set $\mathcal{F}_{P, n}^{\tau}=+\infty$ and $\mathcal{F}_{G}^{\tau}=+\infty$.

Step 1 For each $1 \leq n \leq$ Pop, evaluate the objective function by invoking Algorithm 1 with inputs $x_{n}^{\tau}$ and $\mathcal{S}$. Assign the output of Algorithm 1 to $\mathcal{F}_{x, n}^{\tau}$.

Step 2 Perform the following assignments. Here $n^{*}=\underset{1 \leq n \leq P o p}{\operatorname{argmin}} \mathcal{F}_{P, n}^{\tau+1}$.

$$
\begin{aligned}
\mathcal{F}_{P, n}^{\tau+1}=\min \left\{\mathcal{F}_{x, n}^{\tau}, \mathcal{F}_{P, n}^{\tau}\right\}, & P_{n}^{\tau+1}=\left\{\begin{array}{ll}
P_{n}^{\tau} & \text { if } \mathcal{F}_{x, n}^{\tau} \geq \mathcal{F}_{P, n}^{\tau} \\
x_{n}^{\tau} & \text { otherwise }
\end{array} \quad \forall 1 \leq n \leq\right. \text { Pop } \\
\mathcal{F}_{G}^{\tau+1}=\min \left\{\mathcal{F}_{G}^{\tau}, \mathcal{F}_{P, n^{*}}^{\tau+1}\right\} & G^{\tau+1}= \begin{cases}G^{\tau} & \text { if } \mathcal{F}_{P, n^{*}}^{\tau+1} \geq \mathcal{F}_{G}^{\tau} \\
P_{n^{*}}^{\tau+1} & \text { otherwise }\end{cases}
\end{aligned}
$$

Step 3 Update the velocities $V_{n}^{\tau+1}$ and the positions $x_{n}^{\tau+1}$ of the particles using the following formulae.

$$
\left\{\begin{array}{l}
V_{n}^{\tau+1}=\omega V_{n}^{\tau}+C_{1} R_{1} \circ\left(P_{n}^{\tau+1}-x_{n}^{\tau}\right)+C_{2} R_{2} \circ\left(G^{\tau+1}-x_{n}^{\tau}\right) \\
x_{n}^{\tau+1}=\mathcal{P}_{X}\left[x_{n}^{\tau}+V_{n}^{\tau+1}\right]
\end{array} \quad \forall 1 \leq n \leq\right. \text { Pop }
$$

where $C_{1}=0.618, C_{2}=0.618$ and $\omega=0.8 . R_{1}$ and $R_{2}$ are two random matrices with proper dimensions; each entry of $R_{1}$ and $R_{2}$ follows a uniform distribution on [0,1]. $\circ$ denotes the Hadamard (entry-by-entry) product. $\mathcal{P}_{X}$ denotes the Euclidean projection onto $X$.

Step 4 If stopping criterion is met (e.g. no improvement in the objective within a given number of consecutive iterations), algorithm terminates. Otherwise, let $\tau=\tau+1$, and go to Step 1 .

This algorithm is applicable to a range of problems with arbitrary spatial-temporal size, rather general junction type and signal specifications, and a wide variety of network performance measures. It can even be easily integrated with commercial traffic simulation softwares by calling them at Step 1 of Algorithm 1. Moreover, it makes no assumption regarding the correlation among different components of the random network inflow vector.

\section{Numerical studies}

This section provides a set of numerical results that illustrate the performance of the proposed LDR-DRO on-line signal control strategy. In particular, we compare the proposed on-line signal control with the traditional fixed-timing signal plan, which serves as a benchmark. The fixed signal plan is based on averaged network inflow profiles obtained from historical data, and produces fixed signal timing plans that are non-responsive to real-time traffic conditions. For our numerical examples, the fixed signal timings are obtained from the mixed integer linear program (MILP) described in Section 3.1.

All optimization and simulation procedures are coded in Matlab interfacing Gurobi ${ }^{4}$ The latter is invoked when a MILP or linear program is solved. All computations are done on the Penn State Lion-X clusters with Intel Xeon X5560 Quad-Core 2.8 GHz and 48Gb Memory. The computations are all sequential with a single thread.

\footnotetext{
${ }^{4}$ Gurobi Optimizer Version 5.5. Gurobi Optimization, Inc., 2013. (software program)
} 


\subsection{Description of the test site and data}

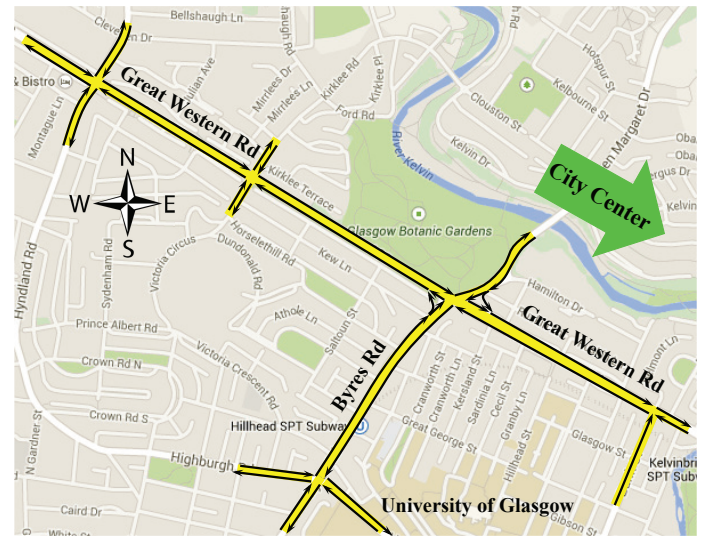

Fig. 2: The test network in west Glasgow.

The numerical experiments are performed for a small portion of the road network on the west end of Glasgow, Scotland and uses empirical data obtained from this location as input. The test network consists of two main corridors, the Great Western Rd and the Byres Rd; see Figure 2. These two corridors are often affected by congestion as they not only connect the radial routes to the city center for drivers approaching from the west, but also provide access to the university and other local major destinations. The portion of the network considered in these tests is highlighted with yellow in Figure 2, and consists of five signalized intersections and 35 directed links.

This case study uses data collected within the CARBOTRAF project, which is funded by the 7th EU Framework (http://www.carbotraf.eu). The historical traffic flow data are obtained from Sky High CountOnUs, a company that provides standard turn-by-turn traffic counts for all the signalized intersections in the test network throughout the years 2007-2009. Since traffic counts collected at various locations were on different dates, these data were converted to the same reference date (June 7, 2010) using scaling factors to avoid biased estimation due to temporal effects on traffic. The scaling factors are derived from the historical Annual Average Daily Flow data at the west end of Glasgow (Transport for Scotland, http://www.transportscotland.gov.uk/map-application), and take into account two sources of flow variation: the seasonality effect and the day-of-the-week effect. The link-specific data are extracted from a coordinated use of map data (UK Ordnance Survey, http://www.ordnancesurvey.co.uk/) and microsimulation conducted within the CARBOTRAF project. The time-dependent vehicle turning percentages are derived from the turn-by-turn traffic counts, which record flows associated with various vehicle movements through an intersection. Our study period spans one hour during the morning peak (8:00-9:00am, June 7, 2010).

\subsection{Generation of synthetic network inflows}

The empirical data described above has insufficient information on the daily variations of the network inflows, and they can only provide the average network inflow profiles. Therefore, we use synthetic traffic flow data that are randomly generated in a way described below. But first, we make note of the fact that even though the underlying distributions of these synthetic data are known a priori in the generation of these synthetic data, our algorithm assumes no such knowledge and relies only on a finite set of samples; that is, our method is guaranteed to perform well with a prescribed statistical significance level, regardless of the underlying distribution of the uncertainties.

We randomly generate a network inflow vector according to the following formula:

$$
\bar{q}_{i}^{t}=\mathcal{P}_{[L, U]}\left[\epsilon_{1, i}^{t}+\epsilon_{2, i}^{t}\right], \quad \forall i \in \mathbb{L}_{i n}, \quad \forall 1 \leq t \leq N
$$

Here, each $\epsilon_{2, i}^{t}, i \in \mathbb{L}_{i n}, 1 \leq t \leq N$, follows a uniform distribution in the range $\left[-\frac{U_{\epsilon}}{2}, \frac{U_{\epsilon}}{2}\right]$ for some given $U_{\epsilon}>0$. Each $\epsilon_{1, i}^{t}, i \in \mathbb{L}_{i n}, 1 \leq t \leq N$, follows one of the specific distributions below: 
1. $\chi^{2}$ distribution: $\epsilon_{1, i}^{t} \sim \chi^{2}\left(v_{i}^{t}\right)$. Here $v_{i}^{t}$ denotes the mean of the distribution, which is given as the average incoming flow for link $i$ at time $t$ from our empirical dataset.

2. Uniform distribution: $\epsilon_{1, i}^{t} \sim U n i\left[0, U_{i}^{t}\right]$. Here $U_{i}^{t}=2 v_{i}^{t}$ is set to be twice the value of the corresponding average inflow from our empirical dataset.

3. Absolute value of a normally distributed random variable: $\epsilon_{1, i}^{t}=\left|\hat{\epsilon}_{1, i}^{t}\right|$, where $\hat{\epsilon}_{1, i}^{t} \sim \mathcal{N}\left(v_{i}^{t}, \sigma^{2}\right)$. Again, the mean $v_{i}^{t}$ is set to be the average inflow of link $i$ at time $t$, and $\sigma^{2}=0.5$.

Finally, $\mathcal{P}_{[L, U]}[\cdot]$ denotes the Euclidean projection onto the set $[L, U]$ to ensure feasibility of the incoming flows.

\subsection{The mixed integer linear programming approach}

In order to test the exact MILP approach, we consider only part of the Great Western Rd corridor, which includes four signalized intersections and 12 directed links; see Figure 3. This network has 5 incoming links; that is, $\mathbb{L}_{\text {in }}=$ $\{1,5,6,7,11\}$. We choose three different time periods, 200, 400, and 800 seconds, and a time step of 10 seconds.

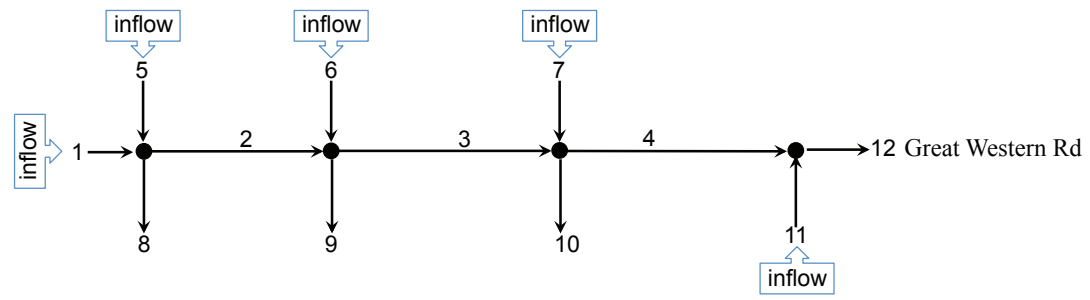

Fig. 3: The test network for the mixed integer linear programming approach, which contains part of the Great Western Rd corridor.

In order to demonstrate our data-driven approach presented in Sections 2.3 and 3.2, we describe here in detail the calibration result for the uncertainty set. In this example $\epsilon_{1, i}^{t}$ follows the second type of distribution (uniform distribution), and $U_{\epsilon}=0.1$ for the uniform distribution of $\epsilon_{2, i}^{t}$. Five out of the 30 sampled time-varying incoming flows (sample paths) for link 1 are shown in Figure 4a, together with the average incoming flow of these 30 samples. It can be seen that the flow variations are substantial. We then apply (11) with $K=30$ samples to construct three candidate sets for the cumulative distribution function (CDF), based on three different significance levels $\alpha \in\{0.05,0.3,0.75\}$. The constructed CDF's are shown in Figure 4b, from which we see that the generated upper and lower bounds completely contain the true CDF. Moreover, larger significance levels $\alpha$ lead to sharper bounds and less conservative estimation of the true CDF, but are more likely to miss the true CDF. This is consistent with the discussion in Section 2.3. For the numerical test a significance level $\alpha=0.3$ is chosen, and the set of candidate distributions for the entire incoming flow vector is constructed according to (22).

The LDR-DRO problem for the off-line stage of the proposed responsive signal control is solved as a MILP presented in Section 3.2. For comparison, the fixed-timing signal control (benchmark) is also solved based on average network inflow vector and the MILP formulation presented in Section 3.1. In order to test the effectiveness of both signal timing strategies, we create scenarios where the inflows into the network are randomly generated according to Section 5.2. We then implement the two signal strategies and calculate the resulting throughput (calculated as the cumulative number of vehicles leaving the network within the horizon) and the objective value (21). Their average values based on 50 such simulation runs are summarized in Table 1. In this table, " $\chi 2$ ", "Uni" and "absGaus" represent the three types of distributions for $\epsilon_{1, i}^{t}$. Each row of the table corresponds to 50 simulation runs, each with a random network inflow vector. We can see that the proposed on-line signal control strategy brings a significant improvement over the optimal fixed-timing plan in terms of maximizing the network throughput. This is partially because the latter approach, although optimal in an uncertainty-free environment, completely ignores the highly stochastic incoming flows in real time situations, which results in a suboptimal system performance.

In terms of computational effort required by the two-stage responsive signal operation, we notice that the majority of the computation is done off line, and the on-line implementation takes little time as it involves only elementary algebraic operations such as linear transformation and projection. Moreover, although the benchmark method takes 


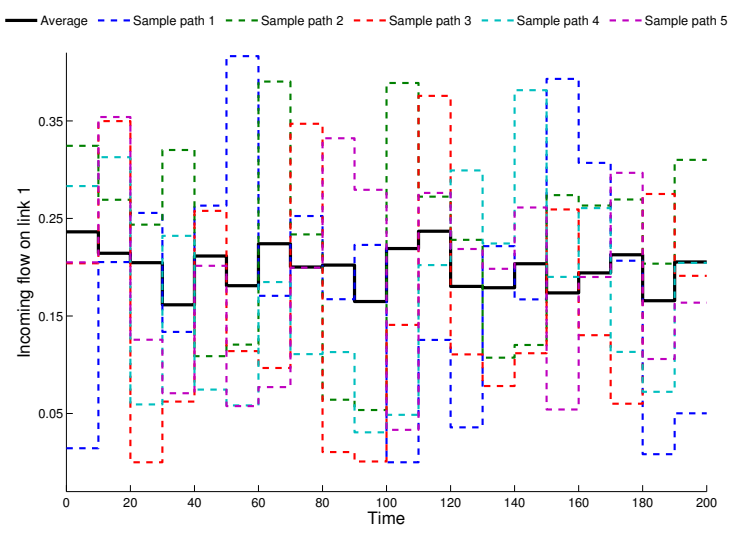

(a)

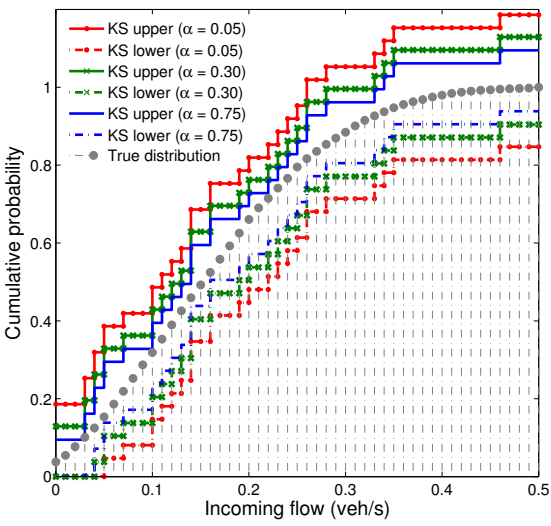

(b)

Fig. 4: Calibrating the uncertainty set of the incoming flow vector. (a) shows the average incoming flow (solid line) of link 1 along with five sampled incoming flows (sample paths) (dashed line). (b) shows the constructed uncertainty sets for the distribution in terms of CDF by invoking (11). "KS upper $(\alpha)$ " and "KS lower $(\alpha)$ " indicate the upper and lower bounds of the confidence region with a given significance level $\alpha \in\{0.05,0.30,0.75\}$.

less time than LDR-DRO in the off-line computation due to its simplistic formulation, it is neither responsive nor robust enough to handle incoming flows observed in real time, and thus is outperformed by our LDR-DRO approach.

Table 1: Comparison between the LDR-DRO approach with the benchmark (fixed-timing) approach. "Throughput" (in vehicles) is the cumulative number of vehicles leaving the network within the time horizon; "Objective" corresponds to the objective function (21); and "CPU time" (in s) is the computational time. "Gap" indicates the relative improvement over the benchmark approach.

\begin{tabular}{|c|c|c|c|c|c|c|}
\hline Distr. of $\epsilon_{1, i}^{t}$ & $U_{\epsilon}$ & Time horizon & Criteria & LDR-DRO & Benchmark & $\operatorname{Gap}(\%)$ \\
\hline$\chi^{2}$ & 0.1 & $200 \mathrm{~s}$ & $\begin{array}{c}\text { Throughput } \\
\text { Objective } \\
\text { CPU time (s) (offline/online) }\end{array}$ & $\begin{array}{c}3.76 \\
0.19 \\
2.66 / 0.00021\end{array}$ & $\begin{array}{c}1.69 \\
0.08 \\
0.18 /-\end{array}$ & $\begin{array}{c}122.5 \% \\
137.5 \% \\
-\end{array}$ \\
\hline Uni & 0.1 & $200 \mathrm{~s}$ & $\begin{array}{c}\text { Throughput } \\
\text { Objective } \\
\text { CPU time (s) (offline/online) }\end{array}$ & $\begin{array}{c}5.82 \\
0.29 \\
3.40 / 0.00028 \\
\end{array}$ & $\begin{array}{c}2.43 \\
0.12 \\
0.29 /- \\
\end{array}$ & $\begin{array}{c}139.5 \% \\
141.7 \% \\
- \\
\end{array}$ \\
\hline absGaus & 0.1 & $200 \mathrm{~s}$ & $\begin{array}{c}\text { Throughput } \\
\text { Objective } \\
\text { CPU time (s) (offline/online) }\end{array}$ & $\begin{array}{c}6.40 \\
0.32 \\
2.68 / 0.00022\end{array}$ & $\begin{array}{c}4.88 \\
0.24 \\
0.32 /-\end{array}$ & $\begin{array}{c}31.1 \% \\
33.3 \% \\
-\end{array}$ \\
\hline absGaus & 0.2 & $400 \mathrm{~s}$ & $\begin{array}{c}\text { Throughput } \\
\text { Objective } \\
\text { CPU time (s) (offline/online) }\end{array}$ & $\begin{array}{c}175.74 \\
6.06 \\
68.17 / 0.00033 \\
\end{array}$ & $\begin{array}{c}163.97 \\
5.59 \\
5.95 /-\end{array}$ & $\begin{array}{c}7.2 \% \\
8.4 \% \\
-\end{array}$ \\
\hline absGaus & 0.2 & $800 \mathrm{~s}$ & $\begin{array}{c}\text { Throughput } \\
\text { Objective } \\
\text { CPU time (s) (offline/online) }\end{array}$ & $\begin{array}{c}519.15 \\
11.90 \\
605.40 / 0.00020\end{array}$ & $\begin{array}{c}473.59 \\
10.35 \\
71.37 /-\end{array}$ & $\begin{array}{c}9.6 \% \\
15.0 \% \\
-\end{array}$ \\
\hline
\end{tabular}

\subsection{The metaheuristic approach}

The test of the metaheuristic approach is conducted on the whole test network shown in Figure 2. We consider a significantly extended time horizon of one hour (8:00-9:00am) with time step of $5 \mathrm{~s}$. In generating random network 
inflow vectors, we employ the third type of distribution for $\epsilon_{1, i}^{t}$, and $U_{\epsilon}=0.1$ for $\epsilon_{2, i}^{t}$. The method of calibrating the uncertain distribution sets remain the same as before.

We again compare the LDR-DRO approach with the fixed-timing plan. We solve the LDR-DRO problem using Algorithm 2, and solve the fixed-timing signal plan using the PSO method instead of MILP, due to the large problem scale and more detailed network specifications. As a third candidate signal plan, we consider the fixed signal timing plan provided by the Glasgow City Council. These fixed timings, including green ratio and offset, are derived to approximate the on-line operation of SCOOT (Split Cycle Offset Optimization Technique) during the morning peak hour in 2010, although they are not exactly the same as SCOOT as the latter is responsive. The numerical results are summarized in Table 2. Again, the performances of the three signal control strategies are averaged over 50 simulation runs. According to this table, the proposed on-line signal control yields a significant improvement over the fixedtiming benchmark approach; it also outperforms the fixed-timing approximation of SCOOT. Not surprisingly, the mass majority of the computational effort is spent on the off-line stage $(24 \mathrm{~h})$, while the on-line implementation only takes $0.01 \mathrm{~s}$. As we have demonstrated, the proposed LDR approach holds promise in supporting real-time signal control decisions in a timely and reliable fashion.

Table 2: Comparison among the proposed on-line signal control, the benchmark timing plan, and the fixed-timing approximation of SCOOT. "Throughput", and "Objective" have the same meanings as in Table 1.

\begin{tabular}{|c|c|c|c|ccc|}
\hline Distr. of $\epsilon_{1, i}^{t}$ & $U_{\epsilon}$ & Time horizon & Criteria & LDR-DRO & Benchmark & SCOOT \\
\hline \hline \multirow{4}{*}{ absGaus } & \multirow{3}{*}{0.1} & \multirow{4}{*}{1 hour } & Throughput & 3910.4 & 1498.3 & 3576.8 \\
& & & Relative gap from LDR-DRO & - & $161.0 \%$ & $9.3 \%$ \\
\cline { 4 - 7 } & & & Objective & 4.2815 & 1.6145 & 4.1439 \\
& & & Relative gap from LDR-DRO & - & $165.2 \%$ & $3.3 \%$ \\
\cline { 4 - 7 } & & & CPU time (offline/online) & $24 \mathrm{~h} / 0.01 \mathrm{~s}$ & $24 \mathrm{~h} /-$ & - \\
\hline
\end{tabular}

\section{Conclusion and future research}

We propose a novel linear decision rule (LDR) approach for on-line signal controls. The LDR enables a fast transition from observed traffic states to signal control variables, which can be realized in real time. To ensure the performance of this LDR in on-line operations, we employ a distributionally robust optimization (DRO) procedure, which takes into account both historical and real-time information. We also propose a data-driven approach for calibrating the set of uncertain distributions to underpin the DRO. Two solution procedures are presented: a mixed integer linear programming approach and a metaheuristic approach. We test the proposed signal control strategies in a simulation environment informed by empirical data from Glasgow, and demonstrate its feasibility in real-time deployment and effectiveness in improving traffic.

We note that the reliability of the LDR-DRO approach is dependent on the quantity and quality of historical data ("training data"), as is the case for almost all statistical learning methods. However, this approach can be applied to a range of scenarios with different levels of data availability, with statistically quantifiable performances derived from our theory. Moreover, the calibration of the uncertainty set can be adjusted to reflect the incorporation of extreme events and/or risk-neutral or risk-averse optimization criteria.

\section{Appendix A. Theoretical results regarding the finite sampling approximation}

This appendix presents theoretical guarantee for the effectiveness of our proposed finite sampling approximation. Theorem 1 below states that the continuous objective can be approximated by the finite sampling approach to an arbitrary degree of precision, by choosing appropriately large sample size $\mathcal{K}$.

Theorem 1. Let the objective of the dual problem (31) be $\tilde{Z}_{2}$ and define its finite sample approximation $\tilde{Z}_{2, \mathcal{K}}$; that is,

$$
\tilde{Z}_{2} \doteq \int_{\mathbb{S}}\left[-\beta_{1}(\boldsymbol{q}) L_{\mathbb{D}}(\boldsymbol{q})+\beta_{2}(\boldsymbol{q}) U_{\mathbb{D}}(\boldsymbol{q})\right] d \boldsymbol{q}+\lambda, \quad \tilde{Z}_{2, \mathcal{K}} \doteq \frac{1}{\mathcal{K}} \sum_{k=1}^{\mathcal{K}}\left[-\beta_{1, k} L_{\mathbb{D}}\left(\boldsymbol{p}^{(k)}\right)+\beta_{2, k} U_{\mathbb{D}}\left(\boldsymbol{p}^{(k)}\right)\right] \prod_{i}^{M}\left(U_{i}-L_{i}\right)+\lambda
$$


with $\beta_{1, k} \doteq \beta_{1}\left(\boldsymbol{p}^{(k)}\right)$ and $\beta_{2, k} \doteq \beta_{2}\left(\boldsymbol{p}^{(k)}\right), \forall k$. Assume that the variance

$$
\sigma_{1}^{2} \doteq \int_{\mathbb{S}} \mathbf{P}(\boldsymbol{q}) \cdot\left[-\beta_{1}(\boldsymbol{q}) L_{\mathbb{D}}(\boldsymbol{q})+\beta_{2}(\boldsymbol{q}) U_{\mathbb{D}}(\boldsymbol{q})\right]^{2} d \boldsymbol{q}-\left[\int_{\mathbb{S}} \mathbf{P}(\boldsymbol{q}) \cdot\left[-\beta_{1}(\boldsymbol{q}) L_{\mathbb{D}}(\boldsymbol{q})+\beta_{2}(\boldsymbol{q}) U_{\mathbb{D}}(\boldsymbol{q})\right] d \boldsymbol{q}\right]^{2}<\infty
$$

where $\mathbf{P}(\boldsymbol{q}) \equiv \frac{1}{\prod_{i}^{M}\left(U_{i}-L_{i}\right)}$ for all $\boldsymbol{q} \in \mathbb{S}$ due to the uniform distribution. Then for an arbitrary $\epsilon>0$, we have

$$
\operatorname{Prob}\left[\left|\tilde{Z}_{2}-\tilde{Z}_{2, \mathcal{K}}\right|<\epsilon\right] \geq 1-\frac{\sigma_{1}^{2} \cdot\left(\prod_{i}^{M}\left(U_{i}-L_{i}\right)\right)^{2}}{\mathcal{K} \epsilon^{2}}
$$

Proof. Since each $\boldsymbol{p}^{(k)}$ is randomly sampled from a uniform distribution in $\mathbb{S}$, we have

$$
\begin{aligned}
E\left[\frac{1}{\mathcal{K}} \sum_{k=1}^{\mathcal{K}}\left[-\beta_{1, k} L_{\mathbb{D}}\left(\boldsymbol{p}^{(k)}\right)+\beta_{2, k} U_{\mathbb{D}}\left(\boldsymbol{p}^{(k)}\right)\right]\right] & =\frac{1}{\mathcal{K}} \sum_{k=1}^{\mathcal{K}} \int_{\mathbb{S}} \mathbf{P}(\boldsymbol{q}) \cdot\left[-\beta_{1}(\boldsymbol{q}) L_{\mathbb{D}}(\boldsymbol{q})+\beta_{2}(\boldsymbol{q}) U_{\mathbb{D}}(\boldsymbol{q})\right] d \boldsymbol{q} \\
& =\int_{\mathbb{S}} \mathbf{P}(\boldsymbol{q}) \cdot\left[-\beta_{1}(\boldsymbol{q}) L_{\mathbb{D}}(\boldsymbol{q})+\beta_{2}(\boldsymbol{q}) U_{\mathbb{D}}(\boldsymbol{q})\right] d \boldsymbol{q} \\
& \operatorname{Var}\left[\frac{1}{\mathcal{K}} \sum_{k=1}^{\mathcal{K}}\left[-\beta_{1, k} L_{\mathbb{D}}\left(\boldsymbol{p}^{(k)}\right)+\beta_{2, k} U_{\mathbb{D}}\left(\boldsymbol{p}^{(k)}\right)\right]\right] \\
= & \frac{1}{\mathcal{K}}\left\{\int_{\mathbb{S}} \mathbf{P}(\boldsymbol{q}) \cdot\left[-\beta_{1}(\boldsymbol{q}) L_{\mathbb{D}}(\boldsymbol{q})+\beta_{2}(\boldsymbol{q}) U_{\mathbb{D}}(\boldsymbol{q})\right]^{2} d \boldsymbol{q}-\left[\int_{\mathbb{S}} \mathbf{P}(\boldsymbol{q}) \cdot\left[-\beta_{1}(\boldsymbol{q}) L_{\mathbb{D}}(\boldsymbol{q})+\beta_{2}(\boldsymbol{q}) U_{\mathbb{D}}(\boldsymbol{q})\right] d \boldsymbol{q}\right]^{2}\right\}
\end{aligned}
$$

According to Chebyshev's inequality, we immediately have, for any $\epsilon_{0}>0$, that

$$
\begin{array}{r}
\operatorname{Prob}\left[\mid \frac{1}{\mathcal{K}} \sum_{k=1}^{\mathcal{K}}\left[-\beta_{1, k} L_{\mathbb{D}}\left(\boldsymbol{p}^{(k)}\right)+\beta_{2, k} U_{\mathbb{D}}\left(\boldsymbol{p}^{(k)}\right)\right]-E\left[\frac{1}{\mathcal{K}} \sum_{k=1}^{\mathcal{K}}\left[-\beta_{1, k} L_{\mathbb{D}}\left(\boldsymbol{p}^{(k)}\right)+\beta_{2, k} U_{\mathbb{D}}\left(\boldsymbol{p}^{(k)}\right)\right] \| \geq \epsilon_{0}\right]\right. \\
\leq \frac{\operatorname{Var}\left[\frac{1}{\mathcal{K}} \sum_{k=1}^{\mathcal{K}}\left[-\beta_{1, k} L_{\mathbb{D}}\left(\boldsymbol{p}^{(k)}\right)+\beta_{2, k} U_{\mathbb{D}}\left(\boldsymbol{p}^{(k)}\right)\right]\right]}{\epsilon_{0}^{2}}
\end{array}
$$

We set $\epsilon_{0}=\frac{\epsilon}{\prod_{i}^{M}\left(U_{i}-L_{i}\right)}$ and derive $\operatorname{Prob}\left[\left|\tilde{Z}_{2}-\tilde{Z}_{2, \mathcal{K}}\right| \geq \epsilon\right] \leq \frac{\sigma_{1}^{2}\left(\prod_{i}^{M}\left(U_{i}-L_{i}\right)\right)^{2}}{\mathcal{K} \epsilon^{2}}$, which is the desired result.

We will next assess the finite approximation (35) of the semi-infinite constraint (32). To do this, we need to first introduce, for constraint (32), a measure of infeasiblity of a solution $\left(x, \lambda, \beta_{1}, \beta_{2}\right)$, denoted by $I_{f}\left(x, \lambda, \beta_{1}, \beta_{2}\right)$ :

$$
I_{f}\left(x, \lambda, \beta_{1}, \beta_{2}\right) \doteq-\int_{\mathbb{S}} \min \left\{-f(x, \boldsymbol{q})+\lambda-\beta_{1}(\boldsymbol{q})+\beta_{2}(\boldsymbol{q}), 0\right\} d \boldsymbol{q},
$$

which is always non-negative. Intuitively, the larger $I_{f}$ is, the more the semi-infinite constraint (32) is violated.

Theorem 2. For the tuple $\left(x, \lambda, \beta_{1}, \beta_{2}\right)$, define $\beta_{1, k} \doteq \beta_{1}\left(\boldsymbol{q}^{(k)}\right), \beta_{2, k} \doteq \beta_{2}\left(\boldsymbol{p}^{(k)}\right), \forall 1 \leq k \leq \mathcal{K}$. Assume $\sigma_{2}^{2} \doteq$ $\int_{\mathbb{S}} \mathbf{P}(\boldsymbol{q})\left[\min \left\{-f(x, \boldsymbol{q})+\lambda-\beta_{1}(\boldsymbol{q})+\beta_{2}(\boldsymbol{q}), 0\right\}\right]^{2} d \boldsymbol{q}-\left[\int_{\mathbb{S}} \mathbf{P}(\boldsymbol{q}) \min \left\{-f(x, \boldsymbol{q})+\lambda-\beta_{1}(\boldsymbol{q})+\beta_{2}(\boldsymbol{q}), 0\right\} d \boldsymbol{q}\right]^{2}<\infty$. If $\beta_{1, k}$ and $\beta_{2, k}$ satisfy constraint (35) for all $1 \leq k \leq \mathcal{K}$, then $\left(x, \lambda, \beta_{1}, \beta_{2}\right)$ satisfies the following: for any $\epsilon>0$,

$$
\operatorname{Prob}\left[I_{f}\left(x, \lambda, \beta_{1}, \beta_{2}\right)<\epsilon\right] \geq 1-\frac{\sigma_{2}^{2}\left[\prod_{i}^{M}\left(U_{i}-L_{i}\right)\right]^{2}}{\mathcal{K} \epsilon^{2}}
$$

Proof. Notice that $\tilde{Z}_{3, \mathcal{K}} \doteq-\frac{1}{\mathcal{K}} \sum_{k=1}^{\mathcal{K}} \min \left\{-f\left(x, \boldsymbol{p}^{(k)}\right)+\lambda-\beta_{1, k}+\beta_{2, k}, 0\right\}$ is a sampling average approximation of $\tilde{Z}_{3} \doteq$ $-\int_{\mathbb{S}} \mathbf{P}(\boldsymbol{q}) \min \left\{-f(x, \boldsymbol{q})+\lambda-\beta_{1}(\boldsymbol{q})+\beta_{2}(\boldsymbol{q}), 0\right\} d \boldsymbol{q}$. Thus, we proceed with an argument similar to that of Theorem 1 by invoking Chebyshev's inequality, and get $\mathbb{P}\left[\left|\tilde{Z}_{3, \mathcal{K}}-\tilde{Z}_{3}\right| \geq \epsilon_{0}\right] \leq \sigma_{2}^{2} /\left(\mathcal{K} \epsilon_{0}^{2}\right)$ for an arbitrary $\epsilon_{0}>0$. If (35) is satisfied, we have $\tilde{Z}_{3, \mathcal{K}}=0$. Therefore, we have $\mathbb{P}\left[\left|\tilde{Z}_{3}\right|<\epsilon_{0}\right] \geq \mathbb{P}\left[\left|\tilde{Z}_{3, \mathcal{K}}-\tilde{Z}_{3}\right|<\epsilon_{0}\right] \geq 1-\sigma_{2}^{2} /\left(\mathcal{K} \epsilon_{0}^{2}\right)$, which immediately leads to the desired result if we let $\epsilon_{0}=\epsilon / \prod_{i}^{M}\left(U_{i}-L_{i}\right)$. 


\section{References}

Banks, A., Vincent, J., Anyakoha, C., 2007. A review of particle swarm optimization. Part I: background and development. Natural Computing 6 (4), 467-484.

Bertsimas, D., Brown, D.B., Caramanis, C., 2011. Theory and applications of robust optimization. SIAM Review 53 (3) $464-501$.

Bertsimas, D., Gupta, V., Kallus, N., 2013. Data-driven robust optimization. arXiv: 1401.0212.

Bertsimas, D., Popescu, I., 2005. Optimal inequalities in probability theory: A convex optimization approach. SIAM Journal on Optimization 15 (3), 780-804

Ceylan, H., Bell, M.G.H., 2004. Traffic signal timing optimisation based on genetic algorithm approach, including drivers routing. Transportation Research Part B 38 (4), 329-342.

Chang, T.-H., Sun, G.-Y., 2004. Modeling and optimization of an oversaturated signalized network. Transportation Research Part B 38 (8), $687-707$.

Chiu, S., Chand, S., 1993. Adaptive traffic signal control using fuzzy logic. In: Proceedings of the Second IEEE International Conference on Fuzzy Systems, pp. 1371-1376.

Christofa, E., Skabardonis, A., 2011. Traffic signal optimization with application of transit signal priority to an isolated intersection. Transportation Research Record: Journal of the Transportation Research Board 2259, 192-201.

Foy, M.D., Benekohal, R.F., Goldberg, D.E., 1992. Signal timing determination using genetic algorithms. Transportation Research Record 1365, $108-115$.

Gayah, V.V., Daganzo, C.F., 2012. Analytical capacity comparison of one-way and two-way signalized street networks. Transportation Research Record 2301, 76-85.

Goh, J., Sim, M., 2010. Distributionally robust optimization and its tractable approximations. Operations Research 58 (4), $902-917$.

Guler, S.I., Cassidy, M.J., 2012. Strategies for sharing bottleneck capacity among buses and cars. Transportation Research Part B 46 (10), 13341345.

Han, K., Gayah, V., Piccoli, B., Friesz, T.L., Yao, T., 2014. On the continuum approximation of the on-and-off signal control on dynamic traffic networks. Transportation Research Part B 61, 73-97.

Han, K., Piccoli, B., Friesz, T.L., Yao, T., 2012. A continuous-time link-based kinematic wave model for dynamic traffic networks. arXiv: $1208.5141 \mathrm{v} 1$

Highway Capacity Manual, 2000. Transportation Research Board, National Research Council, Washington, DC.

Kennedy, J., Eberhart, R., 1995. Particle swarm optimization. Proceedings of IEEE International Conference on Neural Networks (Perth, Australia), IEEE Service Center, Piscataway, NJ.

Lighthill, M., Whitham, G., 1955. On kinematic waves. II. A theory of traffic flow on long crowded roads. Proceedings of the Royal Society of London. Series A, Mathematical and Physical Sciences 229 (1178), 317-345.

Lebacque, J., Khoshyaran, M., 1999. Modeling vehicular traffic flow on networks using macroscopic models, in Finite Volumes for Complex Applications II, 551-558, Hermes Science Publication, Paris, 1999.

Lucas, D.E., Mirchandani, P.B., Head, L.K, 2000. Using remote simulation to evaluate real-time traffic control strategies. Transportation Research Record 1727, 10-15.

Massey, F.J. 1951. The Kolmogorov-Smirnov test for goodness of fit. Journal of the American Statistical Association 46 (253), 68-78.

Miller, A.J., 1963. Computer control system for traffic networks. In: Proceedings of the 2nd International Symposium on Theory of Traffic Flow, London, UK.

Murat, Y.S., Gedizlioglu, E., 2005. A fuzzy logic multi-phased signal control model for isolated junctions. Transportation Research Part C 13 (1), 19-36.

Newell, G. F., 1993. A simplified theory of kinematic waves in highway traffic. Part I: General theory. Transportation Research Part B 27 (4), 281-287.

Richards, P.I., 1956. Shockwaves on the highway. Operations Research 4 (1), $42-51$.

Robertson, D.J., Bretherton, R.D., 1974. Optimal control of an intersection for any known sequences of vehicle arrivals. In: Proceedings of the 2nd IFAC-IFIP-IFORS Sumposium on Traffic Control and Transport Systems, Monte Carlo.

Stephens, M.A., 1974. Edf statistics for goodness of fit and some comparisons. Journal of the American Statistical Association. 69 (347), $730-737$.

Sun, D., Benekohal, R.F., Waller, T.S., 2006. Bi-level programming formulation and heuristic solution approach for dynamic traffic signal optimization. Computer-Aided Civil and Infrastructure Engineering 21 (5), 321-333.

Thas, O., 2010. Comparing distributions. Springer.

Yperman, I., S. Logghe, L. Immers, 2005. The link transmission model: an efficient implementation of the kinematic wave theory in traffic networks, advanced OR and AI methods in transportation, Proc. 10th EWGT Meeting and 16th Mini-EURO Conference, Poznan, Poland, 122-127, Publishing House of Poznan University of Technology.

Zhang, L., Yin, Y., Lou, Y., 2010. Robust signal timing for arterials under day-to-day demand variations. Transportation Research Record 2192, 156-166. 